\title{
Severe COVID-19 infection is associated with aberrant cytokine production by infected lung epithelial cells rather than by systemic immune dysfunction
}

Thomas Gajewski ( $\nabla$ tgajewsk@medicine.bsd.uchicago.edu )

University of Chicago

Sherin Rouhani

University of Chicago

Jonathan Trujillo

University of Chicago

Athalia Pyzer

University of Chicago

Jovian Yu

University of Chicago

https://orcid.org/0000-0001-9214-7576

Jessica Fessler

University of Chicago

Alexandra Cabanov

University of Chicago

Emily Higgs

University of Chicago

Kyle Cron

University of Chicago

Yuanyuan Zha

University of Chicago

Yihao Lu

University of Chicago

Jeffrey Bloodworth

University of Chicago

Mustafa Abasiyanik

University of Chicago

Susan Okrah

University of Chicago

Blake Flood

University of Chicago 


\section{Ken Hatogai}

University of Chicago

\section{Michael Leung}

University of Chicago

\section{Apameh Pezeshk}

University of Chicago

\section{Lara Kozloff}

University of Chicago

\section{Robin Reschke}

University of Chicago https://orcid.org/0000-0002-2850-2526

\section{Garth Strohbehn}

University of Chicago

\section{Carolina Soto Chervin}

University of Chicago

\section{Madan Kumar}

University of Chicago

https://orcid.org/0000-0003-0866-8681

\section{Stephen Schrantz}

University of Chicago

Maria Lucia Madariaga

University of Chicago https://orcid.org/0000-0002-2950-7230

\section{Kathleen Beavis}

University of Chicago

Kiang-Teck Yeo

University of Chicago

\section{Randy Sweis}

University of Chicago

Jeremy Segal

University of Chicago

\section{Savaş Tay}

University of Chicago https://orcid.org/0000-0002-1912-6020

Evgeny Izumchenko

University of Chicago

Jeffrey Mueller

University of Chicago

\section{Lin Chen}

University of Chicago

\section{Article}


Keywords: COVID-19, respiratory failure, severe COVID-19 pathophysiology

Posted Date: November 24th, 2021

DOI: https://doi.org/10.21203/rs.3.rs-1083825/v1

License: (c) (1) This work is licensed under a Creative Commons Attribution 4.0 International License. Read Full License 
Severe COVID-19 infection is associated with aberrant cytokine production by infected lung epithelial cells rather than by systemic immune dysfunction

3 Authors: Sherin J Rouhani ${ }^{+1}$, MD PhD; Jonathan A Trujillo+1, MD PhD; Athalia R Pyzer ${ }^{+1}$, MD PhD; Jovian $4 \mathrm{Yu}^{1}, \mathrm{MD}$; Jessica Fessler ${ }^{2}, \mathrm{PhD}$, Alexandra Cabanov², Emily F Higgs², Kyle R. Cron², Yuanyuan Zha ${ }^{3}, \mathrm{PhD}$;

5 Yihao Lu ${ }^{4}$, Jeffrey C. Bloodworth ${ }^{1}$; Mustafa Fatih Abasiyanik ${ }^{5}$ PhD; Susan Okrah ${ }^{5}$; Blake A Flood PhD;

6 Ken Hatogai ${ }^{1,2}$ MD PhD, Michael YK Leung ${ }^{2}$; Apameh Pezeshk ${ }^{1}$ MD, Lara Kozloff ${ }^{1}$ PhD, Robin Reschke ${ }^{2}$

7 MD; Garth W. Strohbehn ${ }^{1}, \mathrm{MD}$; Carolina Soto Chervin ${ }^{1}, \mathrm{MD}$ PhD; Madan Kumar ${ }^{6}$, DO; Stephen Schrantz ,

8 MD; Maria Lucia Madariaga ${ }^{8}$, MD; Kathleen G Beavis ${ }^{2}$, MD; Kiang-Teck J. Yeo PhD; Randy F. Sweis ${ }^{1}$,

9 MD; Jeremy Segal ${ }^{2} \mathrm{MD}$ PhD, Savaş Tay ${ }^{5} \mathrm{PhD}$, Evgeny Izumchenko ${ }^{1} \mathrm{PhD}$; Jeffrey Mueller ${ }^{2}$, DO, Lin S

10 Chen ${ }^{4}$ PhD; Thomas F Gajewski², MD PhD

\section{Affiliations:}

131 Department of Medicine, Section of Hematology/Oncology, University of Chicago, Chicago, IL

142 Department of Pathology, University of Chicago, 5841 S. Maryland Ave, MC2115, Chicago, IL

153 The Human Immunological Monitoring Facility, University of Chicago, Chicago, IL 60637

164 Department of Public Health Sciences, The University of Chicago, Chicago, IL 60637

175 Pritzker School of Molecular Engineering, University of Chicago, Chicago, IL, USA

186 Department of Pediatrics, Section of Infectious Diseases, University of Chicago

197 Department of Medicine, Section of Infectious Diseases, University of Chicago

208 Department of Surgery, University of Chicago

$21+$ These authors contributed equally to this work

\section{Corresponding Author:}

24 Thomas F. Gajewski, M.D. Ph.D

25 Department of Medicine, University of Chicago, 5841 S. Maryland Ave, MC2115, Chicago, IL, 60637

26 Email:tgajewsk@medicine.bsd.uchicago.edu 


\section{Abstract:}

The mechanisms explaining progression to severe COVID-19 remain poorly understood. It has

29 been proposed that immune system dysregulation/over-stimulation may be implicated, but it is not clear 30 how such processes would lead to respiratory failure. We performed comprehensive multiparameter 31 immune monitoring in a tightly controlled cohort of 128 COVID-19 patients, and used the ratio of oxygen 32 saturation to fraction of inspired oxygen ( $\mathrm{SpO} 2 / \mathrm{FiO} 2)$ as a physiologic measure of disease severity. 33 Machine learning algorithms integrating 139 parameters identified IL-6 and CCL2 as two factors predictive 34 of severe disease, consistent with the therapeutic benefit observed with anti-IL6-R antibody treatment. 35 However, transcripts encoding these cytokines were not detected among circulating immune cells. Rather, 36 in situ analysis of lung specimens using RNAscope and immunofluorescent staining revealed that elevated

37 IL-6 and CCL2 were dominantly produced by infected lung type II pneumocytes. Severe disease was not 38 associated with higher viral load, deficient antibody responses, or dysfunctional T cell responses. These 39 results refine our understanding of severe COVID-19 pathophysiology, indicating that aberrant cytokine 40 production by infected lung epithelial cells is a major driver of immunopathology. We propose that these 41 factors cause local immune regulation towards the benefit of the virus. 


\section{Introduction}

The clinical manifestations of COVID-19 range in severity from asymptomatic infection to critical

44 illness and death, yet the mechanisms by which SARS-CoV-2 cause morbidity and mortality have yet to be

45 fully elucidated. It has been proposed that an excessive immune response may cause immunopathology in

46 affected target organs, particularly the lower respiratory tract. Several large studies of hospitalized patients

47 demonstrated that disease severity and mortality are correlated with elevated levels of inflammatory

48 cytokines, suggesting a potentially dysregulated immune response to infection ${ }^{1-4}$. Consistent with this

49 notion, the steroid dexamethasone improved outcomes in severe and critically ill patients ${ }^{5,6}$. IL-6 specifically

50 has been proposed as a functionally important cytokine, ${ }^{7}$ and the anti-IL-6R antibody (Ab) tocilizumab

51 provided a survival benefit in critically ill COVID-19 patients ${ }^{8,9}$.

52 SARS-CoV-2-infected patients can develop both T cell and B cell responses ${ }^{2,3,10-12}$. Some groups

53 of patients appear to develop phenotypically distinct immune responses, which have been hypothesized to

54 be maladaptive $\mathrm{e}^{2-4,10,13}$. This includes skewing towards a Th2 or Th17 phenotype ${ }^{2}$ or uncoordinated B and/or

55 T cell responses ${ }^{10}$. However, these studies used heterogeneous cohorts of patients at different phases of

56 infection, and concurrent disease states or immunosuppression may complicate the interpretation of

57 immunologic studies. African American and Latino patients are disproportionately affected by the SARS-

58 CoV-2 pandemic, but they generally are under-represented in translational research studies. Similarly, 59 analysis of non-hospitalized patients with mild COVID-19 has been limited. Based on these considerations, 60 we examined the longitudinal immune response from non-immunosuppressed, predominantly African

61 American and Latino patients. We defined disease severity based on the ratio of oxygen saturation to 62 fraction of inspired oxygen ( $\mathrm{SpO} 2 / \mathrm{FiO} 2)$. Immune parameters associated with disease severity were 63 identified based on an unbiased machine learning algorithm. When a disconnect was identified between 64 elevated serum cytokine levels yet lack of evidence for their production by immune cells, lung tissue was 65 studied for in situ expression of key immune genes. 
$\underline{\text { Results }}$

Patients and definition of disease severity

We analyzed 101 hospitalized COVID-19 patients, 27 non-hospitalized COVID-19 outpatients, and 22 healthy donors (HD) as part of a COVID-19 biobanking protocol (Fig 1a). Sixty-seven additional COVID19 patients were excluded from analysis because of potential immunological confounders, as listed in Supplementary Table 1. Samples from patients who received the anti-IL-6R antibody tocilizumab were excluded from cytokine analyses, as tocilizumab can modulate levels of IL-6 and other cytokines ${ }^{14}$. Demographic characteristics of the study cohort are shown in Supplementary Table 2. Our patient population was $68 \%$ African-American with a median age of 55 years. To avoid over-sampling bias from severe patients who had more timepoints available for analysis, we used the maximum level of soluble factors quantified per patient from an early (Day 1-9) and late (Day 10-30) timepoint post-symptom onset, except when assessing cytokine kinetics.

To obtain an objective measure of disease severity to correlate with immunologic parameters, the pulse oximeter oxygen saturation $\left(\mathrm{SpO}_{2}\right)$ / fraction of inspired oxygen $\left(\mathrm{FiO}_{2}\right)$ ratio $(\mathrm{S} / \mathrm{F}$ ratio) was calculated for each patient over time (Fig 1a). The S/F ratio is analogous to the $\mathrm{PaO}_{2} / \mathrm{FiO} 2(\mathrm{P} / \mathrm{F})$ ratio used in $\mathrm{ARDS}$

81 studies ${ }^{15,16}$ and has been validated as an independent correlate of severity in SARS-CoV-2 infection ${ }^{16}$.

82 Patients were divided into three groups - mild, moderate, and severe - on the basis of their worst daily mean $83 \mathrm{~S} / \mathrm{F}$ ratio during their initial hospitalization. Patients with normal oxygen saturation on room air and 84 outpatients were defined as mild ( $\mathrm{S} / \mathrm{F}>315)$, while the majority of inpatients on non-invasive or invasive 85 ventilatory support were classified as severe (Supplementary Fig 1a). The S/F ratio is a dynamic and objective measurement of a patient's respiratory status over the course of illness and hospitalization

87 (Supplementary Fig 1c) and provides a continuous scale of disease severity.

\section{Robust adaptive immune responses in infected patients}

Consistent with other studies ${ }^{17}$, severe patients had higher maximum C-reactive protein (CRP),

91 ferritin, and D-dimer levels compared to mild patients (Supplementary Fig 1d). The absolute lymphocyte 
One hypothesis potentially explaining disease severity was a diminished or delayed adaptive immune response, leading to failed viral clearance. We therefore measured total immunoglobulin (lg), $\lg$, and IgM antibody titers against the SARS-CoV-2 Spike glycoprotein and its Receptor Binding Domain (RBD). RBD binds to angiotensin-converting enzyme 2 (ACE2) receptor on human cells and is the primary target for neutralizing antibodies ${ }^{18-20}$. By day 10 post-symptom onset, 44 of 45 evaluable patients had detectable anti-Spike and anti-RBD total Ig (Figure 1b). IgG titers to Spike and RBD persisted through the acute phase of infection (day 10-19), recovery (day 20-59), and into late recovery (day 60 - 120), whereas IgM titers started to decline in the recovery phase, as expected (Figure 1c). There was a corresponding increase in the frequency of antibody-producing plasmablasts, class switched $\lg \mathrm{D}^{\text {neg }} \mathrm{B}$ cells, and $\mathrm{T}$ follicular helper cells (Tfh) by day 9 (Fig $1 \mathrm{~d}$ ). When examining Ab responses by disease severity, patients with severe disease developed comparable maximum anti-RBD and anti-Spike antibody titers compared to patients with mild or moderate disease (Figure 1b, 1e), indicating that a failed Ab response was not causal for progression to severe disease. Spike and RBD titers also did not correlate with age (Supplementary Fig $2 a$ ) or gender (Supplementary Fig 2b). IL-6 is known to be involved in plasma cell differentiation and antibody production ${ }^{21,22}$, so we investigated whether treatment with the IL-6R antagonist tocilizumab affected SARS-CoV-2 antibody generation; yet no differences were observed (Supplementary Fig 2c). Antiviral medications such as remdesivir could have decreased antigen load and led to a lesser Ab response; however, no diminution of $A b$ response was observed (Supplementary Fig 2c). Consistent with these results, nasopharyngeal viral load as measured by digital droplet PCR (ddPCR) did not differ between patients with mild, moderate, or severe disease (Supplementary Fig 2d).

Analysis of circulating T cells by flow cytometry (Supplementary Fig 3) revealed a decrease in 114 the percentage of $\mathrm{CD}^{+} \mathrm{T}$ cells relative to total $\mathrm{CD} 45^{+}$cells in severe patients (Fig 2a). The percentages of $115 \mathrm{CD}^{+}$central memory (CM) and effector memory (EM) cells decreased at both early and late time points, 116 while the frequency of terminally differentiated memory (TEMRA) cells was relatively stable (Fig 2a). The 117 percentage of $\mathrm{CD}^{+} \mathrm{CM}$ cells increased in patients while the percentage of $\mathrm{CD} 4^{+} \mathrm{EM}$ cells remained 118 unchanged. The percentage of T helper type 1 (Th1) cells was increased in severe COVID-19 patients while the percentage of T helper type 2 (Th2) cells did not change. CD4 ${ }^{+} \mathrm{T}$ cells upregulated CD57, a 
marker of cytotoxic terminally differentiated cells, ${ }^{23,24}$ and CD8 ${ }^{+}$T cells upregulated CD95 (Supplementary 121 Fig 4a,4b).

122 CD38 and HLA-DR are markers of activated T cells during viral infections ${ }^{25}$, and this population 123 was increased among both $\mathrm{CD}^{+}$and CD8 ${ }^{+} \mathrm{T}$ cells in COVID-19 patients of all disease severities (Fig 2b). 124 It was particularly striking in $\mathrm{CD}^{+} \mathrm{T}$ cells, where $42 \%(21 / 50)$ of patients had $>5 \%$ of all $\mathrm{CD} 8^{+} \mathrm{T}$ cells 125 expressing these activation markers between days 10-30. Thus, despite a relative overall lymphopenia, 126 there was an abundance of activated T cells in severe patients. Self-clustering analysis using UMAP and 127 FlowSOM algorithms ${ }^{26}$ showed COVID-19 patients had increased percentages of activated CD8 ${ }^{+}$(cluster 128 4) and $\mathrm{CD}^{+}$(cluster 11) cells expressing high levels of CD38, HLA-DR, and CD95 (Supplementary Fig 4c129 e). $\mathrm{CD}^{+} \mathrm{CM}$ cells and multiple subsets of $\mathrm{CD}^{+} \mathrm{T}$ cells upregulated CD28 (Supplementary Fig 4a,4b). 130 There were no significant differences in the percentages of $\mathrm{CD} 4^{+}$or $\mathrm{CD} 8^{+} \mathrm{T}$ cells expressing PD-1, although 131 modest upregulation of PD-1 was seen on $\mathrm{CD}^{+} \mathrm{EM}$ and $\mathrm{CD}^{+} \mathrm{EM}$ and TEMRA cells (Fig 2c, 132 Supplementary Fig 4a,b). There was an increased percentage of CD8 ${ }^{+} \mathrm{T}$ cells expressing TIM-3 (Fig 2c). 133 The proportion of regulatory T cells (Tregs) also increased in COVID-19 patients (Fig 2a), suggesting a counter-regulatory mechanism in response to increasing levels of T cell activation.

Several studies have shown that inhibitory receptors including PD-1 are upregulated on SARSCoV-2 specific T cells, and have suggested that PD-1 ${ }^{\text {high }}$ cells in COVID-19 infection are exhausted ${ }^{17,27-29}$ or have decreased polyfunctionality ${ }^{28,30}$. However, PD-1 can also be upregulated in acutely activated T 138 cells $^{3,31}$. To determine whether there were differences in IFN- $\gamma$ production by SARS-CoV-2-specific T cells, we used an ELISPOT to measure IFN-y production after stimulation with overlapping HLA class I \& II 15mer peptides from the S, M, and N proteins of SARS-CoV-2. IFN-Y production was seen as early as day 8 141 after symptom onset, and the degree of IFN-Y production was similar between patients with different 142 disease severities (Supplementary Fig 4f).

143 To determine if PD-1 on these cells represents a marker of activation or exhaustion, we used 144 intracellular cytokine staining to measure polyfunctionality after S/M/N peptide stimulation. Compared to 145 mild patients, severe patients had higher percentages of polyfunctional CD4 ${ }^{+} \mathrm{T}$ cells producing IFN- $\mathrm{\gamma}$, TNF$146 \alpha$, and/or IL-2 in response to S/M/N peptide stimulation (Fig 2d-f). Furthermore, cytokine production was 147 concentrated in the PD $-1^{+} \mathrm{CD} 4^{+} \mathrm{T}$ cells, indicating that PD-1 represents an activation marker rather than a 
marker of dysfunction in this context. There was a similar trend with PD- $1^{+} \mathrm{CD} 8^{+} \mathrm{T}$ cells in severe patients, but this was not significant due to increased patient-to-patient variability in the $\mathrm{CD}^{+} \mathrm{T}$ cell response (Supplementary Fig 4g). Cytokine-producing T cells were enriched amongst the CD38+HLA-DR ${ }^{+}$population

(Fig $2 \mathrm{~d}, 2 \mathrm{f}$ ), consistent with this population containing virus-activated T cells. We conclude that the adaptive immune response is robust in severe COVID-19 patients and that lack of virus-specific immunity is not contributory to the progression to disease severity.

Patients with SARS-CoV-2 also had a serum cytokine and chemokine profile consistent with increased T cell activation. Levels of CCL19 and CCL20, which recruit T cells to lymph nodes for activation, increased with disease severity (Fig $2 \mathrm{~g}$ ). Severe patients had higher levels of SCD25/IL-2Ra, which is cleaved and released upon T cell activation. CCL5 and CXCL10 recruit T cells to sites of inflammation, and were elevated in the serum of COVID-19 patients. CXCL10 also increased with disease severity. Levels of CCL19, CCL20, and CD25/IL-2Ra remained elevated over time in severe patients, while CCL5 and CXCL10 levels declined over time in both mild and severe patients (Fig $2 \mathrm{~h}$ ). Patients with severe disease had increased levels of T cell survival cytokines IL-15 and IL-7 (Supplementary Fig 4h). Levels of the immunoregulatory molecules IL-10 and IL-1RA were increased in patients with SARS-CoV-2, suggestive of an expected negative feedback loop in response to increasing T cell activation ${ }^{32,33}$.

\section{Innate immune cells and circulating cytokines}

Analysis of the innate immune response demonstrated a decreased proportion of circulating NK cells and particularly the CD16- NK cell subset at early and late timepoints (Fig 3a). Frequencies of dendritic cell (DC) subsets remained mostly unchanged other than a decrease in $\mathrm{CD}_{123^{+}}$plasmacytoid dendritic 169 cells at late timepoints (Supplementary Fig 5a). However, the level of CD86 increased in plasmacytoid DCs, 170 indicating a more activated status. CD1c $\mathrm{C}^{+} \mathrm{DCs}$ also had higher levels of Tim-3 at late time points after 171 SARS-CoV-2 infection. The proportions of neutrophils, non-classical monocytes, and intermediate 172 monocytes were increased in patients with COVID-19 compared to healthy controls (Fig 3a). While the 173 percentage of classical monocytes was unchanged, the mean fluorescence intensity (MFI) of CD86 and 174 HLA-DR was decreased in infected patients (Fig 3a), suggesting the emergence of less-mature monocytes 175 from the bone marrow. This is further supported by increased levels of the myeloid growth factor GM-CSF 
176 in the serum of patients with SARS-CoV-2 infection (Fig 3b), and a negative correlation between GM-CSF 177 and HLA-DR levels on intermediate monocytes $\left(R^{2}=0.15, p=0.004\right)$ (Supplementary Fig $\left.5 c\right)$. These 178 parameters are consistent with tissue repair-type macrophages being favored during SARS-CoV-2 179 infection.

180 Patients with SARS-CoV-2 had increased levels of cytokines responsible for recruiting neutrophils, 181 monocytes and macrophages to sites of inflammation, including the neutrophil chemoattractants IL-8, 182 CXCL1, CXCL2, and the monocyte chemoattractants CCL2, CCL4, and CX3CL1 (Fig 3b). IL-8, CCL2, and 183 CX3CL1 also increased with disease severity. Distinct groups of cytokines clustered together in correlation plots at late time points (Fig 3c), particularly in severe patients. CCL2 levels remained high over time in severe patients, and higher levels of CCL2 also correlated with a longer duration of moderate or severe illness $\left(R^{2}=0.17, p=0.00737\right)($ Fig 3d-e).

IL-6 has been identified as a pathologic mediator of cytokine release syndrome after CAR-T cell treatment, and it has been hypothesized that a similar phenomenon may be driving severe pathology in some COVID-19 patients ${ }^{7}$. IL-6 signals through the IL-6R and gp130 complex. Gp130 is ubiquitously expressed, while IL-6R expression is normally limited to immune cells and hepatocytes. IL-6 can also form a complex with soluble IL-6R (sIL-6R $\alpha)$ and signal in trans through gp130 in cells that do not express the IL-6R. We found that IL-6 levels increased with disease severity, while sIL-6R $\alpha$ and gp130 levels were similar between severity groups (Fig 3b). While there was a correlation between CRP and IL-6 levels, there 194 were many patients who had a high CRP but only a modest increase in IL-6 (Supplementary Fig 5d). Levels 195 of IL-6 remained high at late timepoints in severe patients when compared to mild (Fig 3d), and levels of soluble gp130 were lower in severe patients at late timepoints (Supplementary Fig 5e). slL-6R $\alpha$ levels remained high over time in both mild and severe patients. Interestingly, the duration of moderate or severe 198 disease positively correlated with IL-6 levels and negatively correlated with soluble levels of gp 130 , which 199 is an endogenous inhibitor of IL-6 trans-signaling ${ }^{34-37}$ (Fig 3e, Supplementary Fig 5f).

200 In order to better understand the pathophysiology that differentiates severe patients from mild or 201 moderate patients, we used the Random Forest machine learning algorithm with 3-fold cross-validation to 202 model the impact of 139 defined immune parameters in an unbiased fashion. From the resulting model, the highest importance features were extracted (Figure 3f), and linear regression modeling was used to 
204 determine the relative impact of each feature. At early time points (D1-9), severe patients showed evidence for an active innate immune response (elevated G-CSF, IL-8, and the percentage of neutrophils) as well as an activated T cell response (elevated CXCL10, IL-7, and IL-15). Integrating the data from all phases (D130) of the immune response, a signature suggestive of $T$ cell recruitment and activation with elevated CCL20, CXCL10, and SCD25/IL-2R was evident in severe patients, consistent with the notion that persistence of virus drives a continued T cell response. Additionally, severe patients had elevated levels of

210 the macrophage related factors CCL2 and IL-6, with elevated CCL2 being the overall top-ranked 211 immunological predictor of severe disease.

\section{IL-6 and CCL2 are produced by infected lung epithelial cells}

Elevated levels of serum IL-6 and CCL2 were each associated with and predictive of severe

215 COVID-19 disease. CCL2 is known to recruit macrophages, particularly M2 macrophages, into tissues. A

216 detrimental role for IL-6 has been supported by studies showing improved clinical outcome upon treatment

217 with the anti-IL-6R antibody tocilizumab ${ }^{9}$. Based on prior work studying cytokine-release syndrome in CAR-

218 T cell therapy ${ }^{38,39}$ and IL-6 production in infectious models ${ }^{40-42}$, it has been assumed that IL-6 in COVID-19

219 patients is being produced by macrophages ${ }^{43}$. However, in our cohort examining representative patients

220 having "high" versus "low" serum IL-6 levels at the protein level (Supplementary Fig 6a), no difference in 221 mRNA for either IL-6 or CCL2 was observed among peripheral level blood mononuclear cells 222 (Supplementary Fig 6c-d). This result is consistent with the flow cytometric analysis of circulating 223 monocytes, which indicated an immature and possibly tissue repair phenotype rather than an inflammatory one (Fig 3a). Together, these results suggested that the source of these cytokines might not be immune 225 cells, but rather raised the possibility that virus-infected cells in the lung might be the major source. We 226 therefore examined expression of IL-6 and CCL2 mRNA in lung tissue from a cohort of 10 fatal COVID-19 227 cases listed in Supplementary Table 3. We performed a multispectral immunofluorescence assay 228 combining RNA in situ hybridization (RNA-ISH) for SARS-CoV-2 RNA and IL-6 or CCL2 mRNA, along with 229 protein immunofluorescence (IF) staining to identify the cells of origin. Thyroid transcription factor 1 (TTF1) 230 was used to identify type 2 pneumocytes, and CD45 was utilized to identify leukocytes (Fig 4a, 231 Supplementary Fig 7a). SARS-CoV-2 RNA was detected in all of the autopsy lung specimens. 
232 Unexpectedly, the vast majority of IL-6 transcripts were detected in cells that did not co-stain for the 233 macrophage markers CD68 or the M2 macrophage marker CD163 (Supplementary Fig 6e-f). Interestingly,

234 large numbers of TTF1+ type 2 pneumocytes expressed IL-6 mRNA, with a high percentage of these cells 235 also positive for SARS-CoV-2 RNA (Fig 4a-c). Quantitative analysis showed TTF1 ${ }^{+}$type 2 pneumocytes were the predominant IL-6-expressing cell type, greatly outnumbering CD45+ immune cells (Fig 4b,c).

237 Among the IL-6 positive populations, type 2 pneumocytes relative to CD $45^{+}$cells showed greater IL-6 238 expression on a per cell basis, as indicated by a greater number of TTF $1^{+}$cells with higher mean staining 239 intensity for IL-6 (Fig 4d). Similarly, CCL2 expression was particularly abundant on TTF2 ${ }^{+}$type 2 240 pneumocytes (Supplementary Fig 7a-d). Together these data show that virus-infected lung epithelial cells 241 are the major source of IL-6 and CCL2 in SARS-CoV-2 infected lungs.

\section{Discussion}

244 Here we show that IL-6 and CCL2 are major factors that discriminate severe infection from mild or 245 moderate disease. IL-6 is known to be produced by innate immune cells such as macrophages or dendritic 246 cells, and by non-immune cells such as epithelial cells or fibroblasts. In allergic asthma ${ }^{44,45}$, SARS-CoV$2471^{40}$, influenza ${ }^{41}$, and pneumovirus infection models ${ }^{42}$, IL- 6 has been shown to be produced by macrophages 248 and other myeloid cells, whereas IL-6 can be produced by cultured nasal epithelial cells infected with 249 RSV46,47. In mouse models of CAR-T cell cytokine release syndrome, macrophages and monocytes are 250 the predominant source of IL- $6^{38,39}$, while vascular endothelial cells have also been shown to produce IL-6 251 in CRS autopsy specimens ${ }^{48}$. Our results from human autopsy specimens unexpectedly show that the 252 predominant source of IL-6 and CCL2 in vivo during SARS-CoV-2 infection is from infected epithelial cells. 253 Our data are consistent with scRNA-seq studies of PBMCs from COVID-19 patients that showed a 254 discrepancy between serum cytokine measurements and the cytokine transcripts of CCL2 and IL-6 among 255 PBMCs $^{49-53}$. Large numbers of epithelial pneumocytes co-stained with IL-6 or CCL2 and SARS-CoV-2 RNA 256 probes, implicating direct cytokine induction by the virus. When considering potential mechanisms of 257 cytokine production, it has been demonstrated that SARS-CoV-2 induces Nuclear Factor kappa B (NF-kB) 258 upregulation and IL-6 production in cultured lung alveolar and epithelial cells ${ }^{54,55}$. CCL2 and other 259 inflammatory mediators are also induced via the NF-kB pathway ${ }^{56}$. 
In mouse models of coronavirus infections, sustained CCL2 expression enhanced the lethality of disease, and promoted immunopathology with a destructive monocyte/macrophage response and ineffective virus clearance ${ }^{57}$. The effect of excess CCL2 in human SARS-CoV-2 has not yet been elucidated. CCL2 may recruit wound-healing M2 macrophages, which can facilitate lung tissue repair by stimulating type 2 pneumocyte expansion ${ }^{58}$, thereby triggering a process capable of enhancing virus propagation ${ }^{59}$. The anti-IL-6R antibody tocilizumab improves survival in critically ill patients ${ }^{9}$, implying that excessive IL-6 is detrimental to the host. Elevated levels of IL-6 in cancer models have been mechanistically linked to decreased DC survival and activation, and consequently impaired $\mathrm{CD} 8^{+} \mathrm{T}$ cell priming ${ }^{60}$. As such, elevated IL-6 expression in the lung during SARS-CoV-2 infection might impair DC function within the infected lung. Thus, we speculate excess IL-6 and CCL2 may favor the virus by promoting a local defect in DC priming of $T$ cells and impaired reactivation of virus-specific T cells locally within the lung and/or by supporting the survival and expansion of infected lung epithelial cells via recruitment of M2 wound-healing macrophages.

Corticosteroids, which improve survival for COVID-19 patients requiring supplemental oxygen ${ }^{5}$, 274 exert their anti-inflammatory effects through NF-KB inhibition and other pathways ${ }^{61}$. It has been assumed 275 that steroids are acting on immune cells, but they could also be inhibiting NF-kB in infected epithelial cells 276 or other host cells. Together, these observations suggest a model whereby SARS-CoV-2 induces NF-kB, leading to an increase in IL-6 and CCL2 production in type 2 pneumocytes, creating favorable conditions

278 for viral persistence, alveolar damage, and ultimately respiratory failure. Additional studies are needed to determine the impact of lung-derived IL-6 and CCL2 on immune clearance of SARS-CoV-2.

Robust adaptive immune responses were seen in patients with mild and moderate disease and were even higher in patients with severe disease, arguing that the lack of a protective immune response did not cause severe disease. CD38 ${ }^{+} \mathrm{HLA}-\mathrm{DR}{ }^{+} \mathrm{CD} 4^{+}$and $\mathrm{CD} 8^{+} \mathrm{T}$ cells were polyfunctional, and patients with a higher disease severity had more cytokine producing $\mathrm{CD}^{+} \mathrm{T}$ cells. These cytokines were being 284 produced by PD $-1^{+}$cells, indicating that PD-1 in this context is a marker of activation, not exhaustion. This 285 is consistent with other recent work showing that tetramer ${ }^{+}$PD- $1^{+}$, SARS-CoV-2-specific CD8 ${ }^{+}$T cells produce cytokines ${ }^{62}$. In patients with severe disease, markers of T cell activation such as SCD25/IL-2R remain high at late time points, suggesting an ongoing immune response against the virus. In some severe 
288 patients who ultimately die from SARS-CoV-2, persistent viral RNA has been demonstrated in longitudinal 289 saliva samples from the Iwasaki group ${ }^{63}$, as well as in our autopsy lung samples. Increased antigen load 290 and duration of antigenic exposure leads to increased T and B cell expansion and differentiation in other 291 experimental models ${ }^{64}$. While we cannot rule out that the increased adaptive immune response causes 292 immunopathology, the increase in regulatory modulators such as IL-10 and Tregs suggests that the immune 293 system is appropriately executing negative feedback pathways. Our data suggests that the immune 294 response to SARS-CoV-2 is a functional and proportional response to infection, and infected pneumocytes 295 are the major source of IL-6 and CCL2, thus revising the paradigm of how we understand the pathogenesis 296 of severe SARS-CoV-2 infection. 
297 Supplementary Table 1: Patients excluded from immune analysis

\begin{tabular}{|l|}
\hline Reasons for exclusion of patients from immune analyses: \\
\hline Trauma/surgery during admission for SARS-CoV-2 \\
\hline Systemic bacterial infection or bacteremia (or high clinical suspicion thereof) \\
\hline Active malignancy \\
\hline $\begin{array}{l}\text { Biologic / Immune Suppressant use within the last } 30 \text { days }>=7.5 \text { mg of prednisone (or within last } 6 \\
\text { months for long-acting monoclonal antibodies). Samples prior to receipt of steroids were eligible for } \\
\text { inclusion. }\end{array}$ \\
\hline Chemotherapy or Immunotherapy within the last 6 months \\
\hline Sickle Cell Disease with crisis within the last 30 days \\
\hline Solid organ transplant \\
\hline Concurrent pneumothorax \\
\hline Saddle pulmonary embolism with hemodynamic compromise \\
\hline Cardiogenic shock in a patient with end stage heart failure; out of hospital cardiac arrest \\
\hline Delivered of a pregnancy during admission for SARS-CoV-2 \\
\hline
\end{tabular}




\begin{tabular}{|c|c|c|c|c|c|c|}
\hline & $\begin{array}{l}\text { Healthy } \\
\text { donor }\end{array}$ & Mild & Moderate & Severe & $\begin{array}{c}\text { Total } \\
\text { SARS- } \\
\text { CoV-2 } \\
\text { positive } \\
\text { cohort }\end{array}$ & $\begin{array}{l}\text { p-value } \\
\text { (mild vs } \\
\text { severe) }\end{array}$ \\
\hline Number of Patients & 22 & 69 & 30 & 29 & 128 & \\
\hline Age; average in years, (range) & $\begin{array}{l}44.9(25- \\
67)\end{array}$ & $\begin{array}{l}53.3(21- \\
>990)\end{array}$ & $\begin{array}{l}53.4(20- \\
79)\end{array}$ & $\begin{array}{l}61.6(35- \\
>90)\end{array}$ & $\begin{array}{l}55.2(20- \\
\quad>90)\end{array}$ & 0.025 \\
\hline Male Sex n (\%) & $12(54.5)$ & $25(36.2)$ & $15(50)$ & $22(75.9)$ & $62(48.4)$ & 7.38 E-04 \\
\hline \multicolumn{7}{|l|}{ Race } \\
\hline Black/African-American n (\%) & $1(4.5)$ & $40(58)$ & $25(83.3)$ & $21(72.4)$ & $87(68)$ & 0.254 \\
\hline White $\mathrm{n}(\%)$ & $16(72.7)$ & $11(15.9)$ & $1(3.3)$ & $4(13.8)$ & $32(25)$ & 1 \\
\hline Hispanic or Latino n (\%) & $3(13.6)$ & $4(5.8)$ & $3(10)$ & $3(10.3)$ & $13(10.2)$ & 0.419 \\
\hline Asian/Mideast Indian n (\%) & $0(0)$ & $3(4.3)$ & $0(0)$ & $0(0)$ & $3(2.3)$ & 0.553 \\
\hline Did not disclose/unknown n (\%) & $2(9.1)$ & $7(10.1)$ & $1(3.3)$ & $1(3.4)$ & $11(8.6)$ & 0.43 \\
\hline More than one Race n (\%) & $0(0)$ & $4(5.8)$ & $0(0)$ & $0(0)$ & $4(3.1)$ & 0.316 \\
\hline $\begin{array}{l}\text { Treated outpatient or discharged } \\
\text { from ED } n(\%)\end{array}$ & --- & $27(39.1)$ & $0(0)$ & $0(0)$ & $27(21.1)$ & $1.31 \mathrm{E}-05$ \\
\hline $\begin{array}{l}\text { Mean LOS for hospitalized } \\
\text { patients; average in days } \\
\text { (range) }\end{array}$ & --- & $5.8(0-27)$ & $8.9(3-25)$ & $16.4(5-56)$ & $9.8(0-56)$ & $1.01 \mathrm{E}-07$ \\
\hline $\begin{array}{l}\text { Died During Index Admission } n \\
(\%)\end{array}$ & --- & $0(0)$ & $0(0)$ & 3 (10.3) & $3(2.3)$ & \\
\hline
\end{tabular}

\section{Comorbidities}

$\begin{array}{lcccccc}\text { CAD or PAD n (\%) } & -- & 7(10.1) & 1(3.3) & 8(27.6) & 16(12.5) & 0.409 \\ \text { COPD or Asthma n (\%) } & --- & 16(23.2) & 7(23.3) & 7(24.1) & 30(23.4) & 0.256 \\ \text { Diabetes n (\%) } & --- & 19(27.5) & 10(33.3) & 18(62.1) & 47(36.7) & 0.337 \\ \text { ESRD n (\%) } & --- & 2(2.9) & 3(10) & 3(10.3) & 8(6.3) & 0.649 \\ \text { HTN n (\%) } & -- & 37(53.6) & 15(50) & 22(75.9) & 74(57.8) & 0.389\end{array}$




$\begin{array}{lcccccc}\text { Azithromycin n (\%) } & --- & 14(20.3) & 9(30) & 14(48.3) & 37(28.9) & 0.626 \\ \text { Lopinavir/Ritonavir n (\%) } & --- & 2(2.9) & 2(6.7) & 0(0) & 4(3.1) & 0.498 \\ \text { Remdesivir n (\%) } & --- & 14(20.3) & 15(50) & 14(48.3) & 43(33.6) & 0.626 \\ \text { Steroid n (\%) } & --- & 0(0) & 0(0) & 1(3.4) & 1(0.8) & 0.451 \\ & & & & & & \\ \text { Tocilizumab* n (\%) } & - & 9(13) & 5(16.7) & 3(10.3) & 17(13.3) & 0.203\end{array}$

300 Significance was calculated using Fisher's Exact Test 

used: AAA - Abdominal aortic aneurysm; CABG - coronary artery bypass graft; CAD - coronary artery

303 disease; CKD - chronic kidney disease; CVA - cerebrovascular accident; DM - diabetes mellitus; HTN 304 hypertension; IDDM - insulin dependent diabetes mellitus; pHTN - pulmonary hypertension; OA 305 osteoarthritis; SLE - systemic lupus erythematosus.

\begin{tabular}{|c|c|c|c|c|c|}
\hline Patient ID & Sex & Age & Race/ethnicity & Comorbidities & $\begin{array}{c}\text { Days } \\
\text { hospitalized } \\
\text { prior to death }\end{array}$ \\
\hline 1 & M & 36 & AA & Obesity, IDDM & 9 \\
\hline 2 & $\mathrm{~F}$ & 50 & Hispanic & $\begin{array}{c}\text { HTN, pHTN, COPD, CAD, } \\
\text { CVA }\end{array}$ & 12 \\
\hline 3 & $\mathrm{~F}$ & 82 & AA & $\begin{array}{c}\text { Alcohol abuse, OA, nursing } \\
\text { home resident }\end{array}$ & 13 \\
\hline 4 & $\mathrm{~F}$ & 68 & AA & $\begin{array}{l}\text { CVA, SLE, C. difficile, } \\
\text { Osteomyelitis }\end{array}$ & 4 \\
\hline 5 & $\mathrm{~F}$ & 84 & $A A$ & $\begin{array}{l}\text { CABG, IDDM, dementia, } \\
\text { breast cancer }\end{array}$ & 4 \\
\hline 6 & M & 84 & AA & $\begin{array}{l}\text { CVA, AAA, prostate cancer, } \\
\text { nursing home resident }\end{array}$ & 1 \\
\hline 7 & M & 40 & AA & Obesity, HTN, IDDM, CAD & 21 \\
\hline 8 & $\mathrm{~F}$ & 77 & AA & HTN, DM, dementia & 15 \\
\hline 9 & $\mathrm{~F}$ & 80 & Caucasian & HTN, IDDM, COPD, CKD & 1 \\
\hline 10 & $\mathrm{~F}$ & 78 & AA & HTN & 4 \\
\hline
\end{tabular}


310 We would like to thank Marcellus Johnson, Melanie Veron, and Lauren Wall for clinical research support, 311 Hongyuan Jiao, PhD, Glee Guilan Li, and Shuhan Yu, MD for biobank sample processing, as well as 312 Rajlakshmi Krishnamurthy MD and Geneatra Green for operational support. Thanks to Laura Johnson from

313 the flow cytometry core, Melvin Lye from Curiox, and Chris Fleming from Cytek for assistance with assay 314 design. The HIM and flow cytometry cores are supported by the University of Chicago Cancer Center 315 Support Grant P30CA014599, and the Center for Research Informatics and Redcap are supported by the 316 UL1TR000430 from NCATS/NIH. SJR, JY and CSC are supported by the T32 CA009566. JAT is 317 supported by 5K12CA139160-09 from NIH. RR was funded by the German Research Foundation (DFG RE 318 4468/1-1). MLM received funding from NIAID Collaborative Influenza Vaccine Innovation Centers (CIVIC) 319 contract 75N93019C00051. YL and LSC are supported by R01GM108711.

321 Author Contributions:

322 SJR, JAT, ARP, TFG conceived and designed the study. SJR, JAT, ARP, AP, LK, GWS, CSC, MK, SS, 323 and MLM consented and recruited patients. YZ processed and stored patient samples and isolated RNA. 324 SJR, JAT, ARP, JF, KC performed flow cytometric assays; AC and JCB performed ELISAs; AC, JAT, BAF, 325 YZ performed cytokine assays; JAT, KH, RR performed microscopy, MFA and SO performed ddPCR. 326 YSJR, JAT, ARP, JY, JF, AC, EFH, KC, YL, JCB, MFA, SO and BAF analyzed data. JY, EFH, YL, and BAF 327 performed bioinformatic analysis, did statistics, and graphed data. ML, KB, K-TJY, JM provided materials 328 and samples. RS, ST, EI, JS, LC, TFG provided personnel, supervision, and insights. SJR, JAT, ARP, 329 and TFG drafted the manuscript; all authors helped to edit the manuscript. 


\section{References}

333 1. Del Valle, D. M. et al. An inflammatory cytokine signature predicts COVID-19 severity and survival. $334 \quad$ Nat. Med. 26, 1636-1643 (2020).

335 2. Lucas, C. et al. Longitudinal analyses reveal immunological misfiring in severe COVID-19. Nature 584, 463-469 (2020).

3. Mathew, D. et al. Deep immune profiling of COVID-19 patients reveals distinct immunotypes with therapeutic implications. Science vol. 369 eabc8511 (2020).

4. Takahashi, T. et al. Sex differences in immune responses that underlie COVID-19 disease outcomes. Nature 588, 315-320 (2020).

5. RECOVERY Collaborative Group et al. Dexamethasone in Hospitalized Patients with Covid-19 Preliminary Report. N. Engl. J. Med. (2020) doi:10.1056/NEJMoa2021436.

6. WHO Rapid Evidence Appraisal for COVID-19 Therapies (REACT) Working Group et al. Association

7. Zhang, C., Wu, Z., Li, J.-W., Zhao, H. \& Wang, G.-Q. Cytokine release syndrome in severe COVID-

8. WHO Rapid Evidence Appraisal for COVID-19 Therapies (REACT) Working Group et al. Association

9. REMAP-CAP Investigators et al. Interleukin-6 Receptor Antagonists in Critically III Patients with Covid-19. N. Engl. J. Med. 384, 1491-1502 (2021).

10. Rydyznski Moderbacher, C. et al. Antigen-Specific Adaptive Immunity to SARS-CoV-2 in Acute COVID-19 and Associations with Age and Disease Severity. Cell 183, 996-1012.e19 (2020).

11. Dan, J. M. et al. Immunological memory to SARS-CoV-2 assessed for greater than six months after infection. Cold Spring Harbor Laboratory 2020.11.15.383323 (2020) doi:10.1101/2020.11.15.383323.

12. Meckiff, B. J. et al. Imbalance of Regulatory and Cytotoxic SARS-CoV-2-Reactive CD4+ T Cells in COVID-19. Cell 183, 1340-1353.e16 (2020). 
13. Sette, A. \& Crotty, S. Adaptive immunity to SARS-CoV-2 and COVID-19. Cell 0, (2021).

14. Azmy, V. et al. Cytokine Profiles Before and After Immune Modulation in Hospitalized Patients with COVID-19. J. Clin. Immunol. (2021) doi:10.1007/s10875-020-00949-6.

15. Rice, T. W. et al. Comparison of the SpO2/FIO2 ratio and the $\mathrm{PaO} 2 / \mathrm{FIO} 2$ ratio in patients with acute lung injury or ARDS. Chest 132, 410-417 (2007).

16. Garibaldi, B. T. et al. Patient Trajectories Among Persons Hospitalized for COVID-19 : A Cohort Study. Ann. Intern. Med. (2020) doi:10.7326/M20-3905.

17. Song, J.-W. et al. Immunological and inflammatory profiles in mild and severe cases of COVID-19. Nat. Commun. 11, 3410 (2020).

18. Barnes, C. O. et al. SARS-CoV-2 neutralizing antibody structures inform therapeutic strategies. Nature (2020) doi:10.1038/s41586-020-2852-1.

19. Rogers, T. F. et al. Isolation of potent SARS-CoV-2 neutralizing antibodies and protection from disease in a small animal model. Science 369, 956-963 (2020).

20. Garcia-Beltran, W. F. et al. COVID-19-neutralizing antibodies predict disease severity and survival. Cell (2020) doi:10.1016/j.cell.2020.12.015.

21. Fornek, J. L. et al. Critical role for Stat3 in T-dependent terminal differentiation of IgG B cells. Blood 107, 1085-1091 (2006).

22. Kopf, M., Herren, S., Wiles, M. V., Pepys, M. B. \& Kosco-Vilbois, M. H. Interleukin 6 influences

23. Kared, H., Martelli, S., Ng, T. P., Pender, S. L. F. \& Larbi, A. CD57 in human natural killer cells and T-lymphocytes. Cancer Immunol. Immunother. 65, 441-452 (2016).

24. Chattopadhyay, P. K. et al. The cytolytic enzymes granyzme A, granzyme B, and perforin: expression patterns, cell distribution, and their relationship to cell maturity and bright CD57 expression. J.

25. Wang, Z. et al. Clonally diverse CD38+HLA-DR+CD8+ T cells persist during fatal H7N9 disease. Nat. Leukoc. Biol. 85, 88-97 (2009). Commun. 9, 824 (2018).

26. Van Gassen, S. et al. FlowSOM: Using self-organizing maps for visualization and interpretation of 
cytometry data. Cytometry A 87, 636-645 (2015).

27. Diao, B. et al. Reduction and Functional Exhaustion of T Cells in Patients With Coronavirus Disease 2019 (COVID-19). Front. Immunol. 11, 827 (2020).

28. Zheng, M. et al. Functional exhaustion of antiviral lymphocytes in COVID-19 patients. Cellular \& molecular immunology vol. 17 533-535 (2020).

29. De Biasi, S. et al. Marked T cell activation, senescence, exhaustion and skewing towards TH17 in patients with COVID-19 pneumonia. Nat. Commun. 11, 3434 (2020).

30. Chen, G. et al. Clinical and immunological features of severe and moderate coronavirus disease 2019. J. Clin. Invest. 130, 2620-2629 (2020).

31. Chikuma, S. et al. PD-1-mediated suppression of IL-2 production induces CD8+ T cell anergy in vivo. J. Immunol. 182, 6682-6689 (2009).

32. Couper, K. N., Blount, D. G. \& Riley, E. M. IL-10: the master regulator of immunity to infection. J. $400 \quad$ Immunol. 180, 5771-5777 (2008).

401 33. Schmitz, N., Kurrer, M., Bachmann, M. F. \& Kopf, M. Interleukin-1 is responsible for acute lung immunopathology but increases survival of respiratory influenza virus infection. J. Virol. 79, 64416448 (2005).

34. Jostock, T. et al. Soluble gp130 is the natural inhibitor of soluble interleukin-6 receptor transsignaling responses. Eur. J. Biochem. 268, 160-167 (2001).

35. Garbers, C. et al. Inhibition of Classic Signaling Is a Novel Function of Soluble Glycoprotein 130

36. Müller-Newen, G. et al. Soluble IL-6 receptor potentiates the antagonistic activity of soluble gp130 on (sgp130), Which Is Controlled by the Ratio of Interleukin 6 and Soluble Interleukin 6 Receptor*. IL-6 responses. J. Immunol. 161, 6347-6355 (1998).

37. Lamertz, L. et al. Soluble gp130 prevents interleukin-6 and interleukin-11 cluster signaling but not intracellular autocrine responses. Sci. Signal. 11, (2018).

38. Giavridis, T. et al. CAR T cell-induced cytokine release syndrome is mediated by macrophages and

415 39. Norelli, M. et al. Monocyte-derived IL-1 and IL-6 are differentially required for cytokine-release 
syndrome and neurotoxicity due to CAR T cells. Nat. Med. 24, 739-748 (2018).

40. Channappanavar, R. et al. Dysregulated Type I Interferon and Inflammatory Monocyte-Macrophage Responses Cause Lethal Pneumonia in SARS-CoV-Infected Mice. Cell Host Microbe 19, 181-193 (2016).

41. Matsukura, S., Kokubu, F., Noda, H., Tokunaga, H. \& Adachi, M. Expression of IL-6, IL-8, and RANTES on human bronchial epithelial cells, NCl-H292, induced by influenza virus A. J. Allergy Clin. Immunol. 98, 1080-1087 (1996).

42. Percopo, C. M. et al. Critical Adverse Impact of IL-6 in Acute Pneumovirus Infection. J. Immunol. 202, 871-882 (2019).

43. Coperchini, F., Chiovato, L. \& Rotondi, M. Interleukin-6, CXCL10 and Infiltrating Macrophages in COVID-19-Related Cytokine Storm: Not One for All But All for One! Front. Immunol. 12, 668507 (2021).

44. Gubernatorova, E. O. et al. Non-redundant Functions of IL-6 Produced by Macrophages and

46. Das, S. et al. Cytokine amplification by respiratory syncytial virus infection in human nasal epithelial Dendritic Cells in Allergic Airway Inflammation. Front. Immunol. 9, 2718 (2018).

45. Marini, M., Vittori, E., Hollemborg, J. \& Mattoli, S. Expression of the potent inflammatory cytokines, cells. Laryngoscope 115, 764-768 (2005).

47. Xie, X.-H. et al. Lipopolysaccharide induces IL-6 production in respiratory syncytial virus-infected airway epithelial cells through the toll-like receptor 4 signaling pathway. Pediatr. Res. 65, 156-162 (2009).

48. Obstfeld, A. E. et al. Cytokine release syndrome associated with chimeric-antigen receptor T-cell therapy: clinicopathological insights. Blood 130, 2569-2572 (2017).

49. Wilk, A. J. et al. A single-cell atlas of the peripheral immune response in patients with severe COVID19. Nat. Med. 26, 1070-1076 (2020). patients. Science 369, 718-724 (2020). 
51. Arunachalam, P. S. et al. Systems biological assessment of immunity to mild versus severe COVID19 infection in humans. Science 369, 1210-1220 (2020).

446 52. Laing, A. G. et al. A dynamic COVID-19 immune signature includes associations with poor prognosis. Nat. Med. 26, 1623-1635 (2020).

53. Zhu, L. et al. Single-Cell Sequencing of Peripheral Mononuclear Cells Reveals Distinct Immune Response Landscapes of COVID-19 and Influenza Patients. Immunity 53, 685-696.e3 (2020).

54. Huang, J. et al. SARS-CoV-2 Infection of Pluripotent Stem Cell-Derived Human Lung Alveolar Type 2 Cells Elicits a Rapid Epithelial-Intrinsic Inflammatory Response. Cell Stem Cell 27, 962-973.e7 (2020).

55. Patra, T. et al. SARS-CoV-2 spike protein promotes IL-6 trans-signaling by activation of angiotensin II receptor signaling in epithelial cells. PLoS Pathog. 16, e1009128 (2020).

56. Ueda, A. et al. NF-kappa B and Sp1 regulate transcription of the human monocyte chemoattractant protein-1 gene. J. Immunol. 153, 2052-2063 (1994).

57. Trujillo, J. A., Fleming, E. L. \& Perlman, S. Transgenic CCL2 expression in the CNS results in a dysregulated immune response and enhanced lethality after coronavirus infection. J. Virol. (2012) doi:10.1128/JVI.03089-12.

58. Lechner, A. J. et al. Recruited Monocytes and Type 2 Immunity Promote Lung Regeneration following Pneumonectomy. Cell Stem Cell 21, 120-134.e7 (2017).

59. Nikolaidis, N. M. et al. Mitogenic stimulation accelerates influenza-induced mortality by increasing susceptibility of alveolar type II cells to infection. Proc. Natl. Acad. Sci. U. S. A. 114, E6613-E6622 (2017).

60. Lin, J. H. et al. Type 1 conventional dendritic cells are systemically dysregulated early in pancreatic carcinogenesis. J. Exp. Med. 217, (2020).

61. Auphan, N., DiDonato, J. A., Rosette, C., Helmberg, A. \& Karin, M. Immunosuppression by glucocorticoids: inhibition of NF-kappa B activity through induction of I kappa B synthesis. Science 270, 286-290 (1995).

62. Rha, M.-S. et al. PD-1-expressing SARS-CoV-2-specific CD8+ T Cells Are Not Exhausted, but Functional in Patients with COVID-19. Immunity 0, (2020). 
472 63. Silva, J. et al. Saliva viral load is a dynamic unifying correlate of COVID-19 severity and mortality. 473 bioRxiv (2021) doi:10.1101/2021.01.04.21249236.

474 64. Blair, D. A. et al. Duration of antigen availability influences the expansion and memory differentiation $475 \quad$ of T cells. J. Immunol. 187, 2310-2321 (2011).

476 65. Amanat, F. et al. A serological assay to detect SARS-CoV-2 seroconversion in humans. Nat. Med.

$477 \quad 26,1033-1036(2020)$.

478 66. Guthmiller, J. J. et al. SARS-CoV-2 infection severity is linked to superior humoral immunity against 479 the spike. bioRxiv (2020) doi:10.1101/2020.09.12.294066.

480 67. Abasiyanik, M. F. et al. Sensitive detection and quantification of SARS-CoV-2 in saliva. Sci. Rep. 11, $481 \quad 12425(2021)$.

482 68. Wei, T. et al. Package 'corrplot'. Statistician (2017).

483 69. Liaw, A. \& Wiener, M. Classification and regression by randomForest. cogns.northwestern.edu.

484 70. Zeileis, A. \& Croissant, Y. Extended Model Formulas in R: Multiple Parts and Multiple Responses.

$485 \quad$ Journal of Statistical Software, Articles 34, 1-13 (2010).

486 71. Aphalo, P. J. ggpmisc: Miscellaneous Extensions to 'ggplot2'. (2021).

487 72. Kassambara, A. ggpubr: 'ggplot2’ Based Publication Ready Plots. (Github).

488 73. Ahlmann-Eltze, C. ggsignif: Significance Brackets for 'ggplot2'. (2019).

489 74. Auguie, B. \& Antonov, A. gridExtra: miscellaneous functions for 'grid' graphics. R package version 2 , $490 \quad$ (2017).

491 75. Harrell, F. E., Jr, from Charles Dupont, W. C. \& others., M. Hmisc: Harrell Miscellaneous. (2020).

492 76. Grolemund, G. \& Wickham, H. Dates and Times Made Easy with lubridate. Journal of Statistical

493 Software vol. $401-25$ (2011).

494 77. Bache, S. M. \& Wickham, H. magrittr: A Forward-Pipe Operator for R. (2020).

495 78. Pedersen, T. L. patchwork: The Composer of Plots. (2020).

496 79. Wickham, H. \& Bryan, J. readxl: Read Excel Files. (2019).

497 80. Ushey, K., Allaire, J. J. \& Tang, Y. reticulate: Interface to 'Python'. (2020).

498 81. Kassambara, A. rstatix: Pipe-Friendly Framework for Basic Statistical Tests. (2020).

499 82. Wickham, H. \& Seidel, D. scales: Scale Functions for Visualization. (2020). 
83. Wickham, H. stringr: Simple, Consistent Wrappers for Common String Operations. (2019).

84. Therneau, T. M. A Package for Survival Analysis in R. https://CRAN.R-project.org/package=survival (2020).

85. Wickham, H. et al. Welcome to the tidyverse. Journal of Open Source Software vol. 41686 (2019).

86. Ooms, J. writexl: Export Data Frames to Excel 'xlsx' Format. (2020).

505 87. Zeileis, A. \& Grothendieck, G. zoo: S3 Infrastructure for Regular and Irregular Time Series. Journal of Statistical Software vol. 14 1-27 (2005).

507 Methods:

508 Patients, sample collection

509 The COVID-19 biobank study was approved by the University of Chicago Institutional Review 510 Board (IRB 20-0520), and all procedures were performed in accordance with the ethical standards set forth 511 in the 1964 Helsinki Declaration. Informed consent was obtained using paper consent, or a Redcap 512 electronic consent form (when possible) to minimize risk of infection. Patients could choose to donate fresh 513 research blood samples and/or allow leftover material from clinical testing (BMP and nasopharyngeal 514 swabs) to be used for research. Research samples were used for flow cytometry, ELISPOTs, and luminex 515 analysis; ELISAs used both research plasma samples and leftover serum from clinical BMPs. Viral load 516 was measured on leftover viral transport media from clinical nasopharyngeal swabs. Blood was collected 517 from inpatients every 24-72 hours during the first week of their hospitalization, and 1-2 times a week for the 518 remainder of their hospitalization. Blood was collected up to once a week from outpatients during regularly 519 scheduled appointments. Serum from 5 healthy donors was purchased from Cellular Technology Limited; 520 the remainder of the healthy donor samples were obtained from the COVID-19 biobank.

$521 \quad$ For serum, fresh blood was collected into a preservative-free vacutainer tube and allowed to clot 522 for at least 30 minutes at room temperature. Leftover plasma samples collected in heparinized tubes were 523 also obtained from the clinical chemistry lab. Tubes were centrifuged for 20 minutes at $1300 \times \mathrm{g}$ at room 524 temperature, and the yellow serum/plasma layers were collected and stored at $-80 \mathrm{deg}$ C until analysis.

525 For PBMCs, fresh blood was collected into heparinized vacutainer tubes and separated using 526 LeucoSep (Greiner Bio-One) tubes. Cells were washed twice with PBS, counted and resuspended in 
527 freezing media containing $90 \%$ FBS and $10 \%$ DMSO at $5-20 \times 10^{\wedge} 6 / \mathrm{mL}$. Cells were frozen in liquid nitrogen 528 until further analysis.

Timepoints used

For analyses divided into early (D0-9) and late (D10-30) phases, the maximal cytokine measurement per patient and the mean of any available flow cytometry measurements per patient in each

533 time period were used. For kinetic analysis of cytokines or antibodies versus DPSO, all available measurements between D1-30 per patient were used. For cytokines versus total non-mild days, the maximal cytokine value between D1-30 was used. Total non-mild days was calculated as the number of

536 hospitalized days with an average $S / F$ ratio of $<315$. For maximal antibody titers, the highest antibody titer measurement between D10-30 per patient were used. Maximum viral load per patient was used. ICS samples were from D11-17 DPSO.

Cytokine measurements by Luminex

Human XL Cytokine Luminex Performance Panel Premixed Kit (CCL2/JE/MCP-1, CCL3/MIP-1

542 alpha, CCL4/MIP-1 beta, CCL5/RANTES, CCL19/MIP-3 beta, CCL20/MIP-3 alpha, CX3CL1/Fractalkine,

543 CXCL1/GRO alpha/KC/CINC-1, CXCL2/GRO beta/MIP-2/CINC-3, CXCL10/IP-10/CRG-2, EGF, G-CSF,

544 GM-CSF, Granzyme B, IFN-alpha, IFN-gamma, IL-1 alpha/IL-1F1, IL-1 beta/IL-1F2, IL-1ra/IL-1F3, IL-2, IL-

545 6, IL-7, IL-10, IL-13, IL-15, IL-33, TGF-alpha, TNF-alpha, TRAIL/TNFSF10, VEGF) and human 6plex

546 luminex multiple kits (angiopoietin-2, CD25/IL-2R alpha, gp130, IL-6R alpha, VEGF-c) were purchased from

547 Bio-Techne, and performed according to manufacturer's instructions. Samples were run in duplicate. Any

548 analytes with a bead count $<32$ or CV $>20 \%$ between the duplicates was considered to fail QC for that 549 patient and excluded from further analysis.

Anti-SARS-CoV-2 antibody ELISA

The SARS-CoV-2 full-length Spike and Receptor Binding Domain (RBD) protein expression constructs were obtained from Florian Krammer and Patrick Wilson ${ }^{65}$, and used to generate recombinant 554 protein for an enzyme-linked immunosorbent assay (ELISA) adapted from established protocols ${ }^{66}$. 
555 Recombinant proteins were produced using a Chinese hamster ovary $(\mathrm{CHO})$ cell line expression system 556 and purified using metal-chelate affinity chromatography. Protein integrity was confirmed via SDS-PAGE 557 gel. Overnight, 96-well ELISA plates (Nunc MaxiSorp high protein-binding capacity plate; ThermoFisher) 558 were coated at $4^{\circ} \mathrm{C}$ with $2 \mathrm{mg} / \mathrm{mL}$ of Spike or RBD protein suspended in Phosphate Buffered Saline (PBS) $\mathrm{pH}$ 7.4. Plates were blocked with $3 \%$ milk powder in PBS containing $0.1 \%$ Tween-20 for 1 hour at room

560 temperature. Serum and plasma samples were heated at $56^{\circ} \mathrm{C}$ for 30 minutes to inactivate virus prior to 561 use. Serial 1:3 dilutions of the samples were prepared in 1\% milk in 0.1\% PBS-Tween 20, and incubated 562 in duplicate with the blocked plate for 2 hours at room temperature. After 3 washes in $0.05 \%$ PBS-Tween 56320 , an HRP-conjugated secondary antibody specific for either total Ig (goat anti-human immunoglobulin Ig, 564 SouthernBiotech), IgM (anti-human IgM, $\mu$-chain specific, Millipore Sigma), or IgG (Goat anti-human IgG $565(\mathrm{H}+\mathrm{L})$, Jackson ImmunoResearch) were diluted in 1\% milk in 0.1\% PBS-Tween-20, and added at 1:8000 566 for 1 hour at room temperature. Plates were washed 3 times with $0.1 \%$ PBS-Tween 20 before being 567 developed with 3, 3', 5, 5'-tetramethylbenzidine (TMB) substrate kit (ThermoFisher) at room temperature. 568 The reaction was stopped after 15 minutes with $2 \mathrm{M}$ sulfuric acid. The optical density (OD) was read at 450 569 nm using a Synergy H4 plate reader (BioTek). The OD values for each sample were background subtracted. 570 A positive control standard was prepared from plasma samples pooled from 6 COVID-19-infected patients, 571 while plasma from an uninfected individual was used as a negative control standard. To account for 572 variability between plates, the OD values were divided by the OD from the negative control from each plate, 573 run at a 1:50 dilution. To quantify the amount of anti-Spike Ig and anti-RBD Ig in the sample, end-point titers 574 were calculated as the linear interpolation of the inverse dilution at which the normalized OD value crossed 575 a threshold of 1 , which was the maximum OD measured for the negative control.

\section{$577 \quad$ Flow cytometry}

578 Frozen PBMCs were thawed into $10 \mathrm{~mL}$ RPMI with 10\% FCS, 1 mM EDTA + DNAse I, washed, 579 and plated on 96 well laminar wash plates (Curiox). Subsequent washes were done using a laminar flow 580 plate washer (Curiox) in a BSL-2 hood, and all staining was done at room temperature. Cells were allowed 581 to settle for 40 minutes, washed, and stained with Live/Dead Blue (Invitrogen) in PBS for 20 minutes. Cells 582 were washed and incubated with monocyte blocker (Biolegend) and CCR7 BV421 (G043H7, Biolegend, 5 
$\mathrm{uL}$ ) for 10 minutes. Brilliant stain buffer plus (BD), CXCR3 PECy7 (G025H7, Biolegend, $5 \mathrm{uL}$ ), CXCR5 BV750 (RF8B2, BD, 1.2 uL), CCR6 BV711 (G034E3, Biolegend, 1.2 uL), and TCRgd PCPCeF710 (B1.1,

585 Thermo, $5 \mathrm{uL}$ ) were added for 10 minutes, and the remainder of extracellular antibodies (CD101 BUV563 586 (V7.1, BD, 5 uL), CD11b PCPCy5.5 (ICRF44, Biolegend, 0.6 uL), CD11c BUV661 (B-ly6, BD, 3.5 uL), CD123 Super Bright 436 (6H6, Thermo, 3.5 uL), CD127 APC-R700 (HIL-7R-M21, BD, 6 uL), CD14 588 SparkBlue550 (63D3, Biolegend, 2.5 uL), CD141 BB515 (1A4, BD, 2.5 uL), CD15 Pacific Orange (VIMC6, 589 Thermo, 5 uL), CD16 BUV496 (3G8, BD, 0.6 uL), CD161 eFluor450 (HP-3G10, Thermo, 5 uL), CD19 SparkNIR685 (HIB19, Biolegend, 2 uL), CD1c AF647 (L161, Biolegend, 5 uL), CD25 PE (BC96, Biolegend, $59110 \mathrm{uL}$ ), CD27 APC (M-T271, Biolegend, 5 uL), CD28 BV650 (CD28.2, Biolegend, 2.5 uL), CD3 BV510 592 (OKT3, Biolegend, 5 uL), CD38 APC-Fire810 (HIT2, Cytek/Biolegend, 1 uL), CD4 CFluor568 (SK3, Cytek, 1.2 uL), CD45 PerCP (2D1, Biolegend, 1.2 uL), CD45RA BUV395 (5H9, BD, 0.3 uL), CD45RO BV605 594 (UCHL1, Biolegend, 5 uL), CD56 BUV737 (NCAM16.2, BD, 3.5 uL), CD57 FITC (HNK-1, Biolegend, 1.2 595 uL), CD8 BUV805 (SK1, BD, 1.2 uL), CD86 BUV615 (BU63, BD, 5 uL), HLA-DR APCF750 (L243, Biolegend, $2.5 \mathrm{uL}$ ), CD95 PECy5 (DX2, Biolegend, 0.6 uL), IgD BV480 (IA6-2, BD, 0.6 uL), IgM BV570 (MHM-88, Biolegend, 2.5 uL), PD-1 BV785 (EH12.2H7, Biolegend, 5 uL), TIM-3 PEDz594 (F38-2E2, Biolegend, $5 \mathrm{uL})$ ) were added for 45 minutes. Cells were washed and resuspended in $2 \%$ paraformaldehyde for 30 minutes, and then washed and run on the Cytek Aurora spectral flow cytometer. Data analyzed using FlowJo.

\section{Intracellular cytokine staining}

Frozen PBMCs were thawed into T cell media (RPMI with 10\% FBS, 2 mM L-glutamine, $1 \mathrm{mM}$

604 sodium pyruvate, $50 \mathrm{uM}$ 2-BME, $100 \mathrm{U} / \mathrm{mL}$ penicillin, $100 \mathrm{mg} / \mathrm{mL}$ streptomycin) washed, and allowed to 605 rest overnight. PepTivator SARS-CoV-2 peptide pools consisting of 15-mer sequences with 11 amino acids overlap against the immunodominant sequence of the surface glycoprotein (“S”), and complete sequences of the membrane glycoprotein ("M") and nucleocapsid phosphoprotein ("N") (all from Miltenyi Biotec) were 608 combined and used at $1 \mathrm{ug} / \mathrm{mL}$, along with $0.5 \mathrm{ug}$ anti-CD28/CD49D antibodies (clone L293/L25, BD biosciences). The PepTivator SARS-CoV-2 peptide pools stimulate both $\mathrm{CD}^{+}$and $\mathrm{CD} 4^{+} \mathrm{T}$ cells. For aCD3/CD28/CD49d, plates were coated overnight at $4 \mathrm{degC}$ with $10 \mathrm{ug} / \mathrm{mL}$ anti-CD3 (clone SK7) in PBS, 
611 and $0.5 \mathrm{ug}$ anti-CD28/CD49d was added into the media with the PBMCs. $0.1 \mathrm{ug} / \mathrm{mL}$ phorbol myristate 612 acetate (PMA) and $1 \mathrm{ug} / \mathrm{mL}$ ionomycin were to the appropriate wells with PBMCs. The unstimulated 613 condition was also treated with 0.5 ug anti-CD28/CD49d. All conditions were incubated for 9 hours in the

614 presence of Golgiplug/Golgistop at manufacturer's recommended concentration (BD Biosciences). After 615 stimulation, $2 \mathrm{mM}$ EDTA was added to the anti-CD3/CD28/CD49d wells and incubated for 15 minutes.

616 Cells were then transferred to a V-bottom 96 well plate for staining. Cells were stained with live/dead blue 617 (Invitrogen) in PBS for 15 minutes prior to adding monocyte blocker and CCR7 BV421 (G043H7, Biolegend, $6185 \mathrm{uL}$ ) for 10 minutes. The remainder of the extracellular antibodies (TCRgd PCPCeF710 (B1.1, Thermo, 5 619 uL), CD3 BV510 (OKT3, Biolegend, 5 uL), PD-1 BV785 (EH12.2H7, Biolegend, 5 uL), CD56 BUV737 620 (NCAM16.2, BD, 3.5 uL), HLA-DR APCF750 (L243, Biolegend, 2.5 uL), CD20 Pacific orange (HI47, 621 Thermo, 5 uL), CD4 CFluor568 (SK3, Cytek, 1.2 uL), CD45 PerCP (2D1, Biolegend, 1.2 uL), CD19 622 SparkNIR685 (HIB19, Biolegend, 2 uL), CD8 BUV805 (SK1, BD, 1.2 uL), CD38 APC-Fire810 (HIT2, 623 Cytek/Biolegend, 1 uL), CD16 BUV496 (3G8, BD, 0.6 uL), CD45RA BUV395 (5H9, BD, 0.3 uL)) and brilliant 624 stain buffer plus (BD) in a total volume of $100 \mathrm{uL}$ were added for 30 minutes, and cells were washed once. 625 Cells were resuspended in the fixation/permeabilization solution from eBioscience's FoxP3 / transcription 626 factor staining buffer for 30 minutes, washed, and incubated with intracellular antibodies in $100 \mathrm{uL}$ (IFNy 627 AF488 (4S.B3, Biolegend, 5 uL), Granzyme AF532 (N4TL33, Thermo, 5 uL), IL-10 APC (JES3-19F1, 628 Biolegend, 5 uL), TNFa BV605 (MAb11, Biolegend, 5 uL), IL-2 BV650 (MQ1-17H12, Biolegend, 5 uL), Ki67 629 Pacific blue (Ki-67, Biolegend, 5 uL), IL-17A PE (BL168, Biolegend, 5 uL), IL-4 PEDz594 (MP4-25D2, 630 Biolegend, 5 uL), IL-6 PECy7 (MQ2-13A5, Biolegend, 5 uL), Perforin BV711 (dG9, Biolegend, 5 uL)) for 60 631 minutes in permeabilization buffer. Cells were washed $2 x$ with permeabilization buffer prior to being 632 resuspended in FACS buffer and run on the Cytek Aurora. Data analyzed using FlowJo. ELISPOT

PBMCs were thawed and washed with $10 \mathrm{~mL}$ RPMI prior to being plated in precoated human

636 IFN-y ELISPOT plates (ImmunoSpot). Control MHC I and II peptide pools against common viral antigens

637 were used as positive controls (MHC I: $2 \mathrm{ug} / \mathrm{mL}$ of CEF peptide pool plus against CMV, EBV, and

638 influenza; MHC II: $50 \mathrm{ug} / \mathrm{mL}$ of CPI pool against CMV, influenza and parainfluenza; both from 
639 ImmunoSpot). Wells with $0.1 \mathrm{ug} / \mathrm{mL}$ PMA and $1 \mathrm{ug} / \mathrm{mL}$ ionomycin were used as an additional positive

640 control. PBMCs in separate wells were stimulated with peptides from either the spike protein ("S"),

641 membrane glycoprotein ("M") or nucleocapsid phosphoprotein ("N") (all from Miltenyi Biotec) at $1 \mathrm{ug} / \mathrm{mL}$.

642 SIYRYYGL (SIY peptide) was used as a negative (irrelevant) peptide control in each experiment with $\leq 1$

643 spot seen in each irrelevant well. The total number of PBMCs recovered after thawing was divided into

64412 wells, with PMA + Ionomycin controls plated at $10 \%$ of the cell density as other wells. Average cell

645 number per experimental well was 218,000 , with a range of 50,000 - 750,000 cells for experimental wells.

646 Cells were incubated at $37^{\circ} \mathrm{C}$ with $5 \% \mathrm{CO} 2$ with activating stimuli for 18 hours in CTL-Test Medium

647 (ImmunoSpot) with 1\% L-glutamine (Gibco) prior to developing plates per manufacturer's recommended

648 procedure. Plates were scanned using ImmunoSpot analyzer and spots were counted using ImmunoSpot

649 7.0.17.0. Spots were normalized by dividing by number of cells plated per well * 100,000 to report spots

650 per 100,000 cells. The sum of the response in the $S+M+N$ wells per 100,000 cells was reported as

651 IFN-Y spots / 100,000 cells.

652

653 Viral load

654 Leftover viral transport media from clinical nasopharyngeal swabs was stored at $-80^{\circ} \mathrm{C}$ until 655 analysis. RNA was extracted using the Qiagen viral RNA mini kit following the manufacturer's instructions, 656 with RNA eluted in $60 \mu \mathrm{L}$ of AVE buffer. Digital droplet PCR (ddPCR) was performed as previously 657 described ${ }^{67}$. Briefly, a $20 \mathrm{uL}$ reaction was performed with $2 \mathrm{uL}$ N1/N2/RNaseP probe primer sets (IDT 658 \#10006770), $5 \mathrm{uL}$ one-step ddPCR supermix, 2 uL reverse transcriptase, $1 \mathrm{uL} 300 \mathrm{mM}$ DTT, and $6 \mathrm{uL}$ 659 sample RNA. Amplification was performed under the following conditions: $25^{\circ} \mathrm{C}$ for $2 \mathrm{~min}, 50^{\circ} \mathrm{C}$ for $60 \mathrm{~min}$, $66095^{\circ} \mathrm{C}$ for $10 \mathrm{~min}, 45$ cycles of $\left[95^{\circ} \mathrm{C}\right.$ for 30 seconds, $55^{\circ} \mathrm{C}$ for $1 \mathrm{~min}$, then $98^{\circ} \mathrm{C}$ for $10 \mathrm{~min}$, followed by 661 infinite hold at $4^{\circ} \mathrm{C}$. Ramping speed was $2.5^{\circ} \mathrm{C} / \mathrm{s}$. Fluorescence was read using a QX200 droplet reader 662 (Bio-Rad) and analyzed with Quantasoft software. Threshold of positivity defined in the manufacturer's FDA 663 emergency use authorization approval was used (>0.1 copy number / $\mu \mathrm{L}$ for either N1 or N2 and more than 6642 positive droplets per reaction). Viral load is reported as the average of the N1 and N2 copy number / $\mu \mathrm{L}$. 665 

hybridization (ISH) using the RNAScope $®$ Multiplex Fluorescent Reagent Kit v2 assay together with 669 antibody-based immunofluorescence staining, according to the manufacturer's integrated co-detection 670 protocol (Advanced Cell Diagnostics, ACD). Briefly, formalin-fixed paraffin-embedded (FFPE) lung tissue sections were baked for $30 \mathrm{~min}$ at $60^{\circ} \mathrm{C}$, deparaffinized by submerging in xylenes for 5 mins twice. The

672 sections were rehydrated in $100 \%$ ethanol for 1 min twice, air dried, treated with RNAScope $®$ hydrogen 673 peroxide for $10 \mathrm{~min}$, and rinsed with distilled water. Target retrieval was performed in a TintoRetriever 674 Pressure cooker (Bio SB) using 1x Co-Detection Target Retrieval solution at $98-102^{\circ} \mathrm{C}$ for $15 \mathrm{~min}$. Slides 675 were rinsed in distilled water and 1x Phosphate-Buffered Saline Tween buffer (PBST). The tissue sections 676 were blocked with Co-Detection antibody diluent (ACD) and incubated with anti-TTF1 antibody (SPT24, 677 BioCare Medical) overnight at $4^{\circ} \mathrm{C}$, washed in PBS-T buffer, incubated in $10 \%$ Neutral Buffered Formalin 678 for 30 min at RT, and washed in PBS-T. Tissue sections were treated with RNAscope ${ }^{\circledR}$ Protease plus at $67940^{\circ} \mathrm{C}$ for $30 \mathrm{~min}$ and rinsed in distilled water. In situ hybridization was performed according to the RNAScope ${ }^{\circledR}$ assay protocol. Briefly, sections were incubated with RNA probe mix and hybridized at $40^{\circ} \mathrm{C}$ for 2 hours. The following RNAscope ${ }^{\circledR}$ probes were used: V-nCoV2019-S-C1 (specific for SARS-CoV-2, S

682 gene encoding the spike protein), Hs-IL-6-C4, Hs-CCL2-C2. Signal amplification was performed using the 683 RNAScope Multiplex FL v2 AMP reagents, AMP1 (30 min, 40 $\mathrm{C}$ ), AMP2 (30 min, 40 $\mathrm{C}$ ), and AMP3 (15 $684 \mathrm{~min}, 40^{\circ} \mathrm{C}$ ), sequentially. Development of the horseradish peroxidase (HRP) signal was performed 685 according to the manufacturer's protocol. Fluorescent labeling of the IL-6 and CCL2 RNA probes was 686 performed using OPAL 620 dye (Akoya Biosciences), while labeling of the SARS-CoV-2 RNA probe was 687 performed using Opal 540 dye. The TTF1 primary antibody was detected with HRP-conjugated secondary 688 antibody (Opal Polymer HRP Ms + Rb, Perkin Elmer) and Opal 690 dye. Subsequent staining on the same 689 sections was performed with an antibody against CD45 (Leukocyte Common Antigen Cocktail: PD7/26/16 690 and 2B11, BioCare Medical) and detected with HRP-conjugated secondary antibody and Opal 520 dye. 691 After all targets were labeled, the sections were incubated with DAPI solution for $5 \mathrm{~min}$ at room temperature 692 and mounted in ProLong Diamond Antifade Mountant (Invitrogen). Co-staining for IL-6 RNA and 693 macrophages was performed using RNAscope $\AA$ probe Hs-IL-6, which was fluorescently labeled with OPAL 694690 dye, and an antibody against either CD68 (Clone KP1, BioCare Medical; diluted in Da Vinci Green 
diluent) or CD163 (Clone 10D6, BioCare Medical; diluted in Renoir Red diluent) and detected with HRPconjugated secondary antibody (Opal Polymer HRP MS+ RB) and OPAL 540 dye. Slides were scanned

697 using the Vectra Polaris imaging platform and Phenochart software (PerkinElmer). For quantitative analysis, 698 up to 200 representative fields of view for each tissue section were acquired at $40 \mathrm{x}$ magnification as multispectral images. Image analysis and cell phenotyping were performed using a supervised machine

700 learning algorithm within the Inform 2.3 software (PerkinElmer), which assigned trained phenotypes and 701 cartesian coordinates to cells.

\section{Clinical data warehouse}

Clinical data was exported through a clinical data warehouse and was also abstracted by a clinical data manager into a standardized RedCap database. Patients with a history of active cancer, organ transplantation were excluded. Medications administered were searched from the clinical data warehouse to find and exclude patients receiving steroids, immunosuppressants, chemotherapy, or immunotherapy agents. Patients with positive blood cultures were identified and excluded. Patients noted to have alternative causes of cardiovascular shock or a pneumothorax were excluded as indicated in Table 1. Outpatients did

710 not routinely have $\mathrm{FiO} 2$ documented, so outpatients were all categorized as mild with an S/F ratio set to 711476 (equivalent to SpO2 100\% on room air).

713 Statistics and Data processing

714 Throughout the paper, boxplots show the medians (middle line) and the first and third quartiles

715 (upper and lower bounds of the boxes). Significance of comparisons from boxplots were determined by

716 two-sided Mann Whitney Wilcoxon test and significance is expressed as p-values, shown as asterisks $\left({ }^{*}\right.$,

$\left.717 \mathrm{p} \leq 0.05 ;^{* *}, \mathrm{p} \leq 0.01 ;{ }^{* * *}, \mathrm{p} \leq 0.001\right)$. All replicates are from distinct samples. Correlations between

718 clinical and research parameters were analyzed using pairwise Spearman's correlation coefficients and

719 were visualized with R package corrplot ${ }^{68}$. Correlation was quantified by a color scale, the significance of 720 the correlation was labeled with asterisks, and boxes with a thick black border represent a false-discovery 721 rate $(\mathrm{FDR})<0.05$. 
722 To determine which features were predictive of disease severity, the Random Forest R package 723 randomForest ${ }^{69}$ was used and area under the ROC curve (AUC) calculated. A 3-fold cross-validation was

724 used and the mean AUC on the test data set was presented. Models were trained for early phase (D0-D9), 725 late phase (D10-D30), and all timepoints (D0-D30) separately. Correlation plots and the machine learning 726 algorithm were processed using $\mathrm{R}$ studio (R 3.6.1).

$727 \quad$ Flow cytometry data were processed using FlowJo V10.7.1. Graphs were created and statistics 728 performed using either GraphPad Prism v9.0.0 or R 4.0.3 (R Core Team, 2020). R packages used include

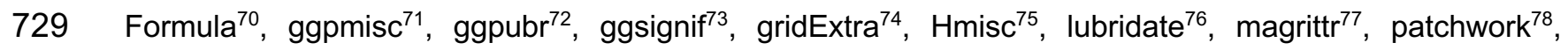
730 readxl $^{79}$, reticulate ${ }^{80}$, rstatix $^{81}$, scales $^{82}$, stringr $^{83}$, survival ${ }^{84}$, tidyverse ${ }^{85}$, writex $\left.\right|^{86}$, zoo $^{87}$, and corrplot $^{68}$.

732 Data Availability

733 Raw .fcs files are available at (will be deposited prior to publication). The datasets generated during 734 and/or analysed during the current study are available from the corresponding author on reasonable 735 request.

736

737 Code Availability

738 Full reproducible code for data processing is available at https://github.com/jovianyu/covid19biobank . 
Figure 2: SARS-CoV-2 infection elicits a robust expansion of activated polyfunctional T cells (a-c) Proportion of immune cell subsets related to adaptive immune responses are shown as a percentage of either live CD45+ cells, live CD4+ cells, or live CD8+ cells, as indicated. Where multiple timepoints within the early (D1-9) or late (D10-30) phase per patient were available, the mean was taken and each patient is represented by one dot per time phase. $n=9$ for HD, $n=15,6,6$ for mild, moderate, and severe respectively in the early phase, and $n=18,15,17$ for mild, moderate, and severe in the late phase. (b, left) Representative flow cytometry plots showing CD38+ HLA-DR+ subsets. (d-f) PBMCs from D11-25 DPSO were stimulated with a combined pool of peptides from the $S, M$ and $N$ proteins for 9 hours and stained for intracellular cytokine production. Background activity in unstimulated wells was subtracted from stimulated wells; negative values after subtraction were set to 0 . Representative flow cytometry plots are shown for TNF $\boldsymbol{\alpha} / \mathrm{IFN}-\mathrm{\gamma}$ (d, upper panels) and TNF $\boldsymbol{\alpha} / \mathrm{PD}-1$ (d, lower panels) staining. The percentage of cells producing various combinations of IFN- $\gamma$, TNF $\alpha$, and IL-2 were reported for CD4+ and CD8+ non-naive (e) and CD4+ and CD8+ CD38+HLADR+ cells (f). (e,f) Comparisons between groups were done by summing the total cytokine production in each column and performing a 2-way ANOVA with Sidak's multiple comparisons test. Error bars represent mean $+/$ - SD for each cytokine subset. $H D n=9$; mild $n=8$; severe $n=7$. Wells with $<50$ CD38+HLA-DR+ cells were excluded from that subset analysis, leaving $n=9 / 7 / 6 \mathrm{HD} / \mathrm{mild} / \mathrm{severe}$ for CD4+CD38+HLA$\mathrm{DR}+$ and $\mathrm{n}=9 / 8 / 6 \mathrm{HD} / \mathrm{mild} / \mathrm{severe}$ for CD8+CD38+HLA-DR+. (g) Peak cytokine and chemokine levels related to T cell activation and survival are shown during the early phase (days 1-9 from symptom onset) and late phase (days 10-30 from symptom onset) of disease in SARS-CoV-2 infection compared to non-infected healthy controls. Each dot represents maximum value per individual subject during each phase of disease (early phase: mild, $n=15$; moderate, $n=10$; severe, $n=11$ and late phase: mild, $n=23$; moderate, $n=16$; severe, $n=19$ and non-infected healthy controls, $n=18$ ). Samples from patients post receipt of tocilizumab (which directly modulates cytokine levels) were excluded from this and subsequent cytokine analyses. (h) Kinetics of cytokine expression over time (days post symptom onset) from mild (green, $\mathrm{n}=33$ ), moderate (orange, $n=19$ ), and severe (red, $n=23$ ) patients. Multiple timepoints per patient plotted when available. Linear regression for cytokine values over time in severe (red) and mild (green) patients shown. Shaded areas represent $95 \%$ confidence interval. (a-c, g) Significance was determined by two-sided Mann Whitney Wilcoxon test and $\mathrm{p}$-values are indicated by asterisks $\left({ }^{*}, p \leq 0.05 ;{ }^{* \star}, p \leq 0.01 ;{ }^{* \star \star}, p \leq 0.001,{ }^{* \star \star *}, p \leq 0.0001\right)$. 
Figure 3: Innate immune changes in SARS-CoV-2 infection (a) Immune cell subsets related to the innate immune response are shown. Boxplots show percentages of each cell population out of live CD45+ cells or MFI of indicated markers. Where multiple timepoints within the early (D1-9) or late (D10-30) phase per patient were available, the mean was taken and each patient is represented by one dot per time phase. $n=9$ for $H D, n=15,6,6$ for mild, moderate, and severe, respectively in the early phase, and $n=18,15,17$ for mild, moderate and severe in the late phase. (b) Peak cytokine and chemokine levels are shown during the early phase (days 1-9 from symptom onset) and late phase (days 10-30 from symptom onset) of disease in SARSCoV-2 infection compared to non-infected healthy controls. Each dot represents maximum value per individual subject during each phase of disease (early phase: mild, $n=15$; moderate, $n=10$; severe, $n=11$ and late phase: mild, $n=23$; moderate, $n=16$; severe, $n=19$ and non-infected healthy controls, $n=18$ ). (c) Correlations between cytokines from days 10-30 for COVID-19 patients were calculated and clustered hierarchically. S/F ratio is fixed as the first column for comparison. Samples were stratified by disease severity. Spearman correlation coefficients were quantified by the scale of color and size of colored squares; significance of the correlation is labeled with * $(P<0.05),{ }^{* *}(P<0.01)$, and ${ }^{* * *}(P<0.001)$. Black border represents a false-discovery rate $(F D R)<0.05$ (d) Kinetics of cytokine expression over time (days post symptom onset) from mild (green, $\mathrm{n}$ $=33$ ), moderate (orange, $n=19$ ), and severe (red, $n=23$ ) patients. Multiple timepoints per patient plotted when available. Linear regression for cytokine values over time in severe (red) and mild (green) patients shown. (e) Peak individual levels of CCL2 and IL-6 are shown as linear correlation with the sum of days each patient spent hospitalized with a moderate or severe S/F ratio, termed "total non-mild days". Each dot represents maximum cytokine value per individual subject; maximal disease severity indicated by color (moderate [orange], $n=20$; severe [red], $n=19$, deceased [black], $n=3$ ). (d-e) Shaded areas represent $95 \%$ confidence interval. (f) The top 10 ranked immune parameters associated with severity per the Random Forest model are tabulated for early phase (days 1-9 from symptom onset) and late phase (days 10-30 from symptom onset) and all time-points (day 1-30 from symptom onset). The linear regression R2 value for each variable is shown in parenthesis, indicating the amount of variation in disease severity that can be explained by this variable alone. $+/$ - denotes direction of association, + indicating the higher the variable the higher (i.e., less severe) the S/F ratio, and - indicating the higher the variable the lower the S/F ratio (i.e. the more severe the disease). Sensitivity, Specificity and AUC are shown. (a-b) Significance was determined by two-sided Mann Whitney Wilcoxon test and $p$-values are indicated by asterisks $\left({ }^{*}, p \leq 0.05 ;{ }^{* *}, p \leq 0.01 ;{ }^{* \star *}\right.$, $\left.\mathrm{p} \leq 0.001,{ }^{* * * *}, \mathrm{p} \leq 0.0001\right)$. 

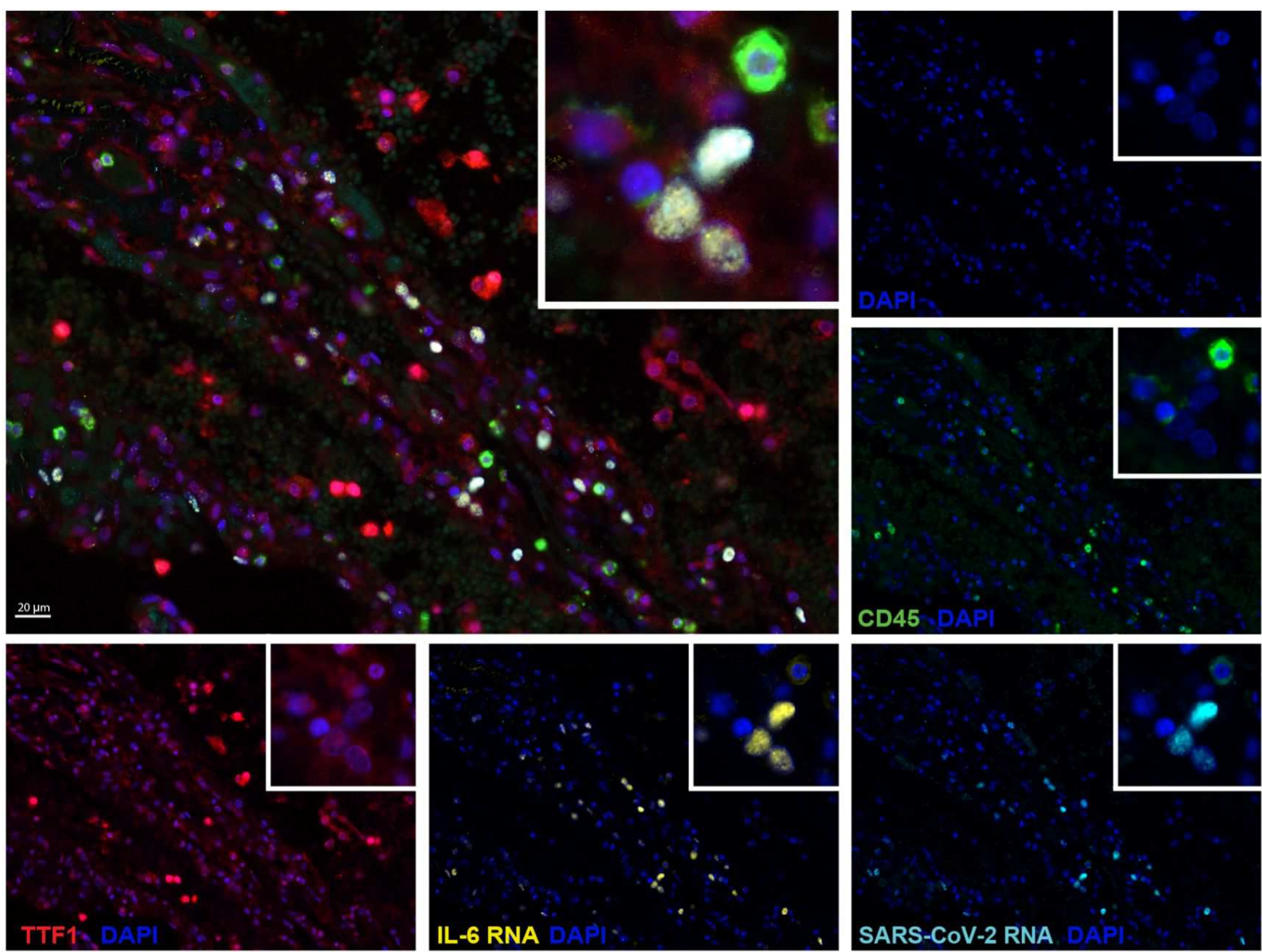

b

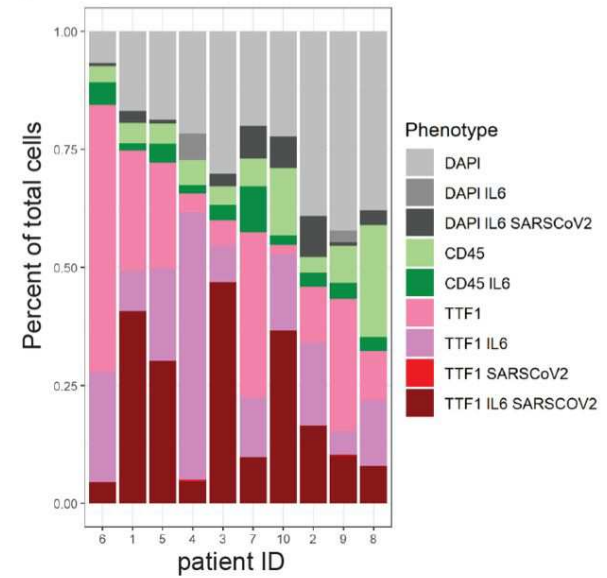

C

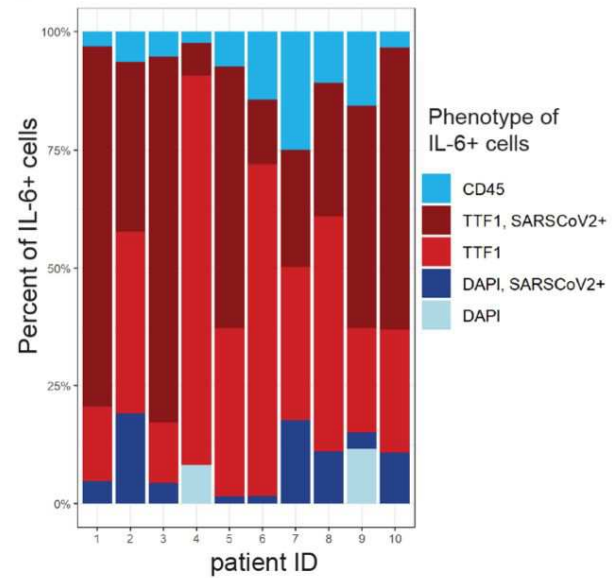

d

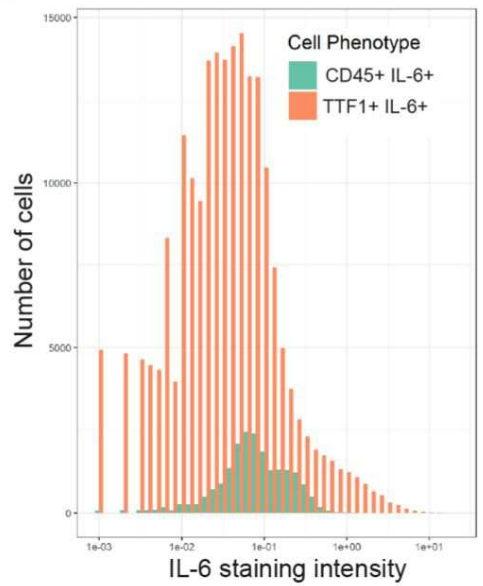

Figure 4: Lung epithelial cells predominantly express IL-6 in lung autopsy tissue in fatal COVID-19. Autopsy lung sections from 10 fatal COVID-19 cases were simultaneously stained for SARS-CoV-2 RNA, IL-6 mRNA, TTF1+ pneumocytes, and CD45+ leukocytes using RNA-ISH combined with multispectral immunofluorescence staining for protein. (a) Representative staining for TTF1 (red), CD45 (green), IL-6 RNA (yellow), SARS-CoV-2 RNA (light blue), and nuclear DAPI counterstain (blue); each stain shown separately and merged. Multispectral images were acquired at 40x magnification. Overlaying high-power images showing SARS-CoV-2 infected TTF1+ pneumocytes expressing high levels of IL-6. (b) Bar plots showing the phenotype composition of cell populations in each autopsy lung specimen. (c) Bar plots showing the phenotype composition of IL-6+ cells in each autopsy lung specimen. (d) Histogram displaying the frequency distribution of mean staining intensity for IL- 6 between TTF1+IL-6+ cells (red) versus CD45+ IL-6+ cells (aqua). Cumulative data from all patients shown. 


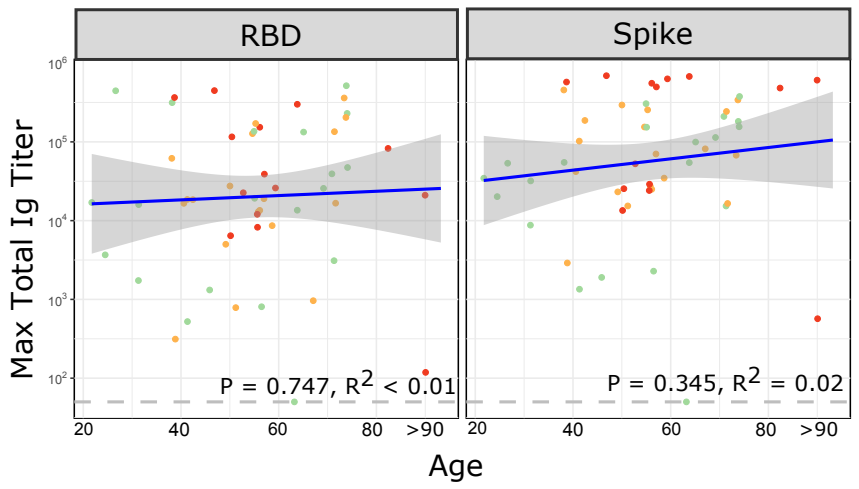

C

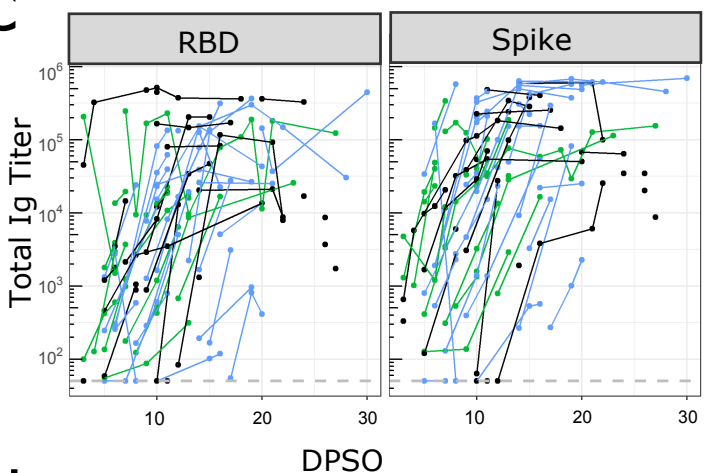

Treatment:

$\rightarrow$ Remdesivir

$\rightarrow-$ Tocilizumab

- Neither
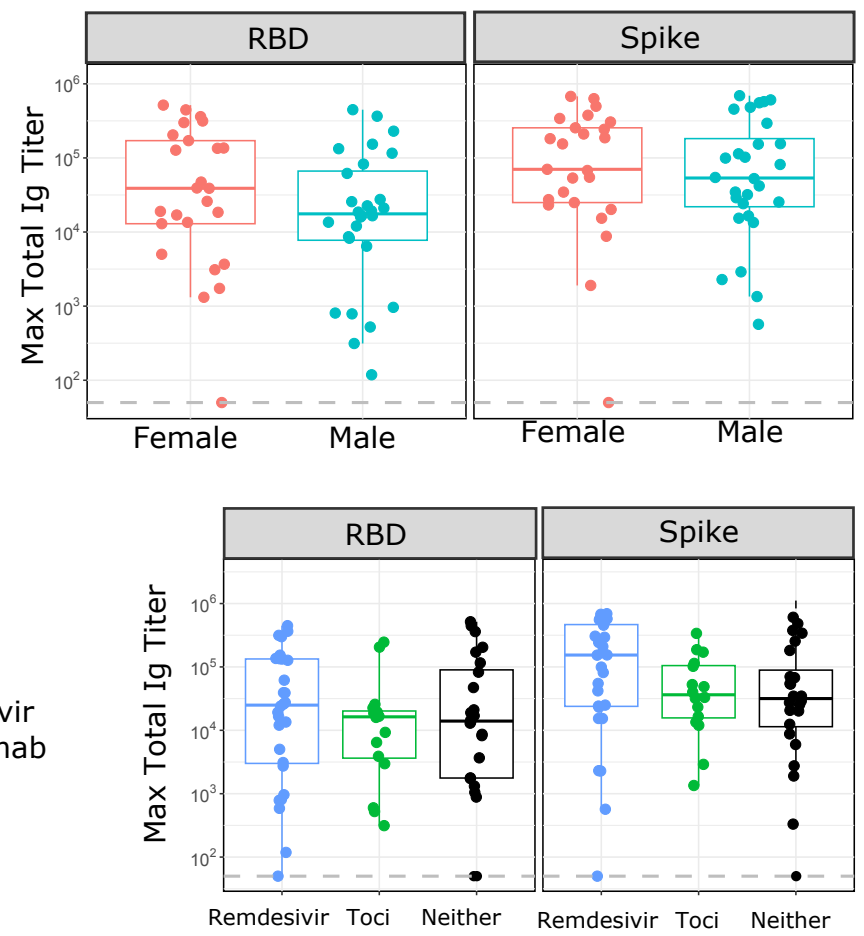

음

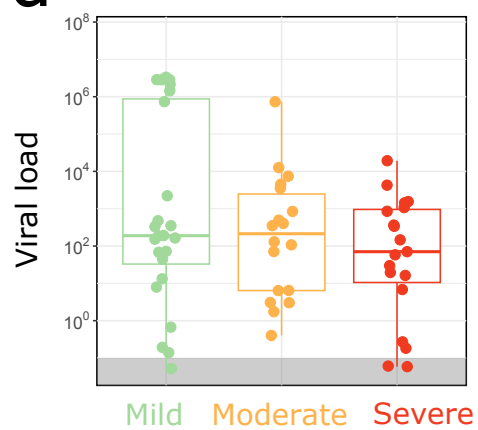

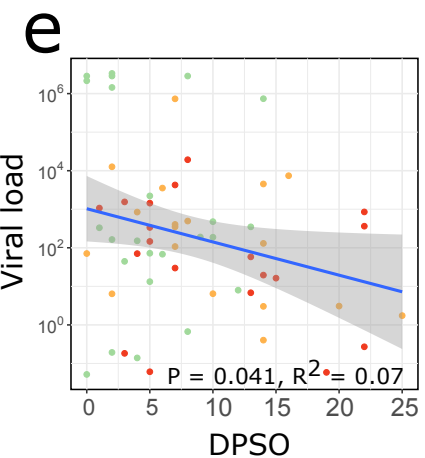

Supplemental Fig 2: Antibody response does not differ based on age, gender, or treatment with remdesivir or tocilizumab (a) Maximum serum SARS-CoV-2 total Ig antibody levels (RBD and Spike) between D10-30 in PCR+ patients $(n=53)$ are shown by age. Disease severity indicated by color (green $=$ mild, orange $=$ moderate, red $=$ severe). (b) Maximum serum SARSCoV-2 total Ig antibody levels (RBD and Spike) between D10-30 in PCR+ patients are shown for females (F, $n=25)$ and males (M, n=28). (c) Serum SARS-CoV-2 total Ig antibody levels (RBD and Spike) in PCR+ patients receiving remdesivir (blue, $n=28$ ), tocilizumab (green, $n=16$ ), or neither (black, $n=24$ ). Antibody levels over time shown in the left panel, and maximum titer per patient shown in the right panel. (d-e) Viral titers were obtained by ddPCR on leftover viral transport medium. Disease severity indicated by color (green = mild, $n=24$; orange $=$ moderate, $n=18$; red = severe disease, $n=19$ ) patients. In d), the grey shaded area denotes negative results. (e) Linear correlation between day post symptom onset and viral load measured by ddPCR, disease severity is indicated by dot color. (a,e) Shaded areas represent $95 \%$ confidence interval. (b,c,d) Groups were compared with two-sided Mann Whitney Wilcoxon test and no significant differences were seen. 


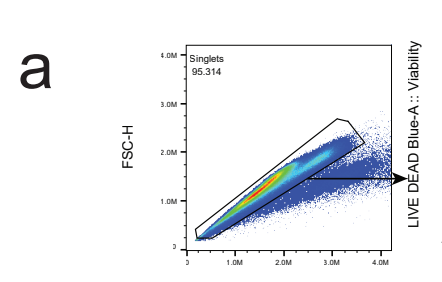

FSC-A

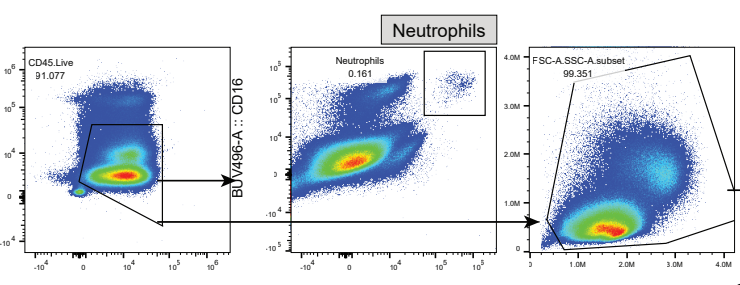

PerCP-A:: CD45

Pacific Orange-A :: CD15
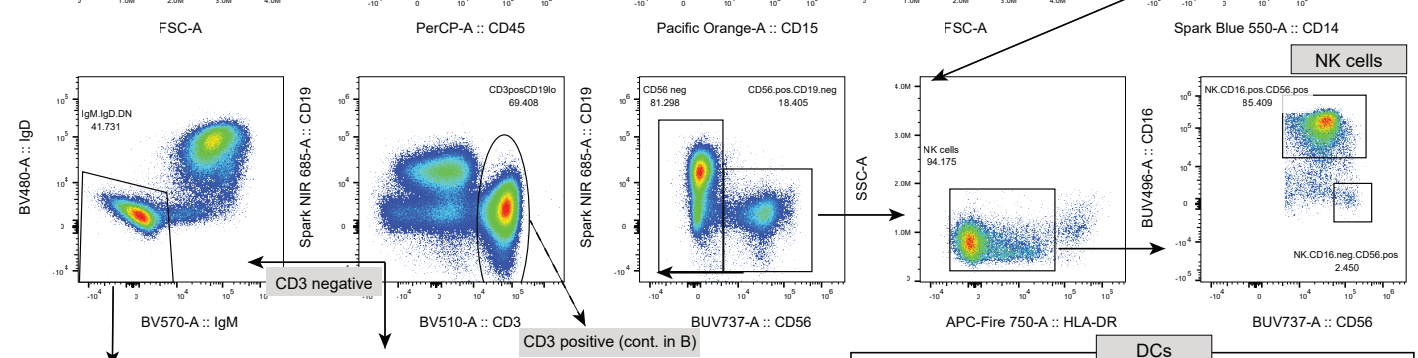

Spark Blue 550-A:: CD14
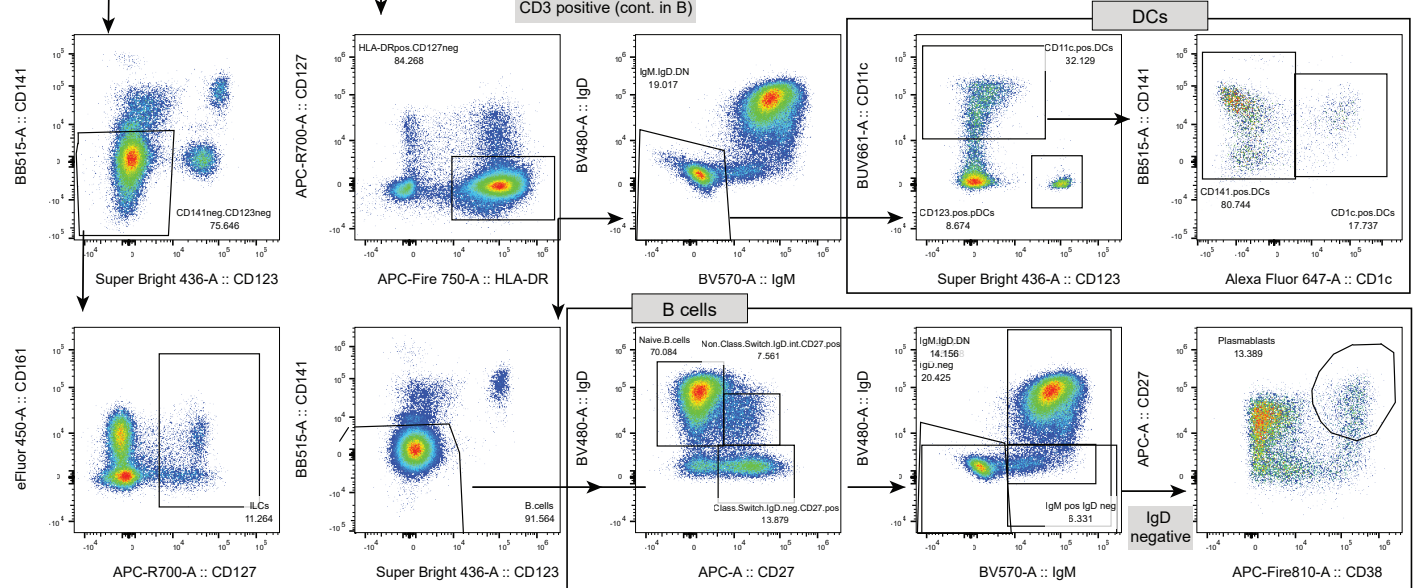

CD3 positive (continued from $A$ )

b
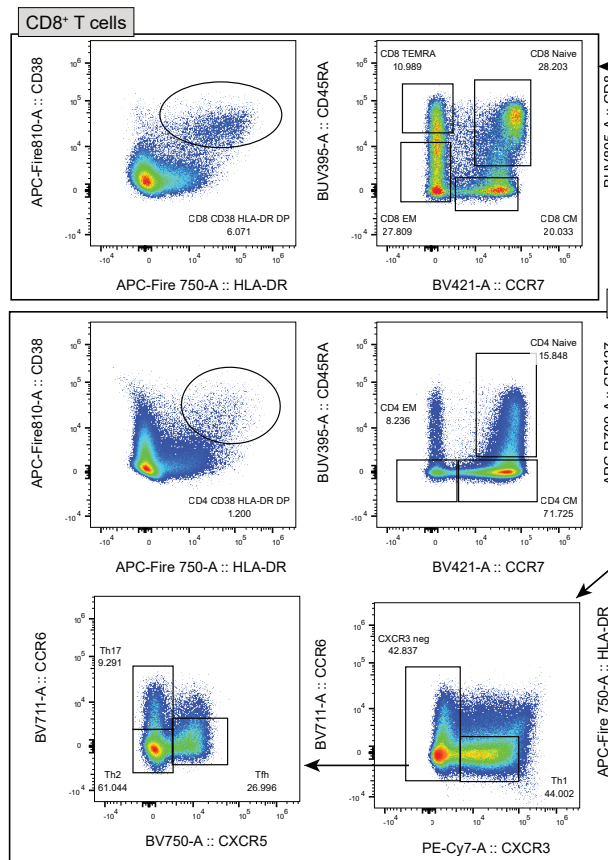

PE-CY7-A :: CXCR3
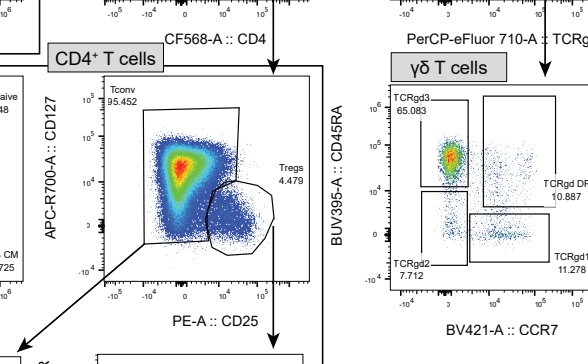

$\bigwedge$
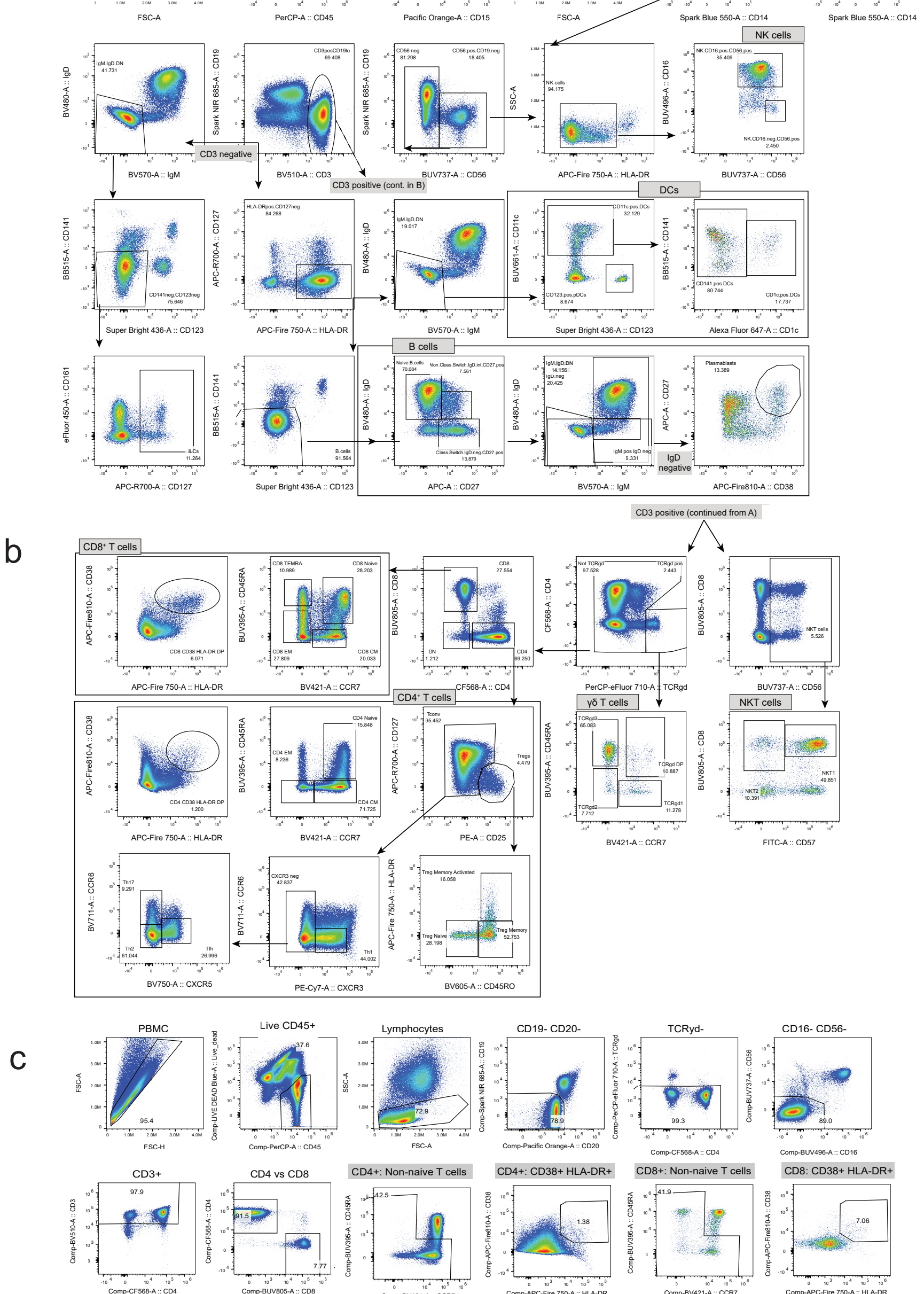

CD4+: Non-naive T cells
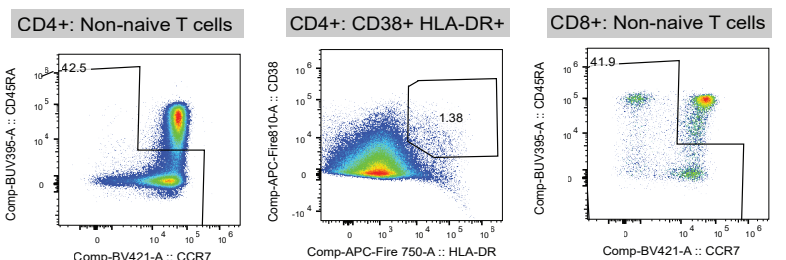

CD8: CD38+ HLA-DR+

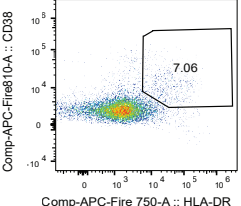

Supplemental Fig. S3 
Supplemental Fig 3: Flow cytometry gating strategy. (a) Gating for extracellular flow cytometry for monocytes, dendritic cells, B cells, and NK cells. (b) Gating for extracellular flow cytometry for T cells, $\gamma \delta$ T cells, and NKT cells. (c) Flow cytometry gating for intracellular cytokine stimulation assays. 

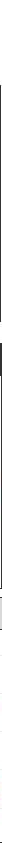
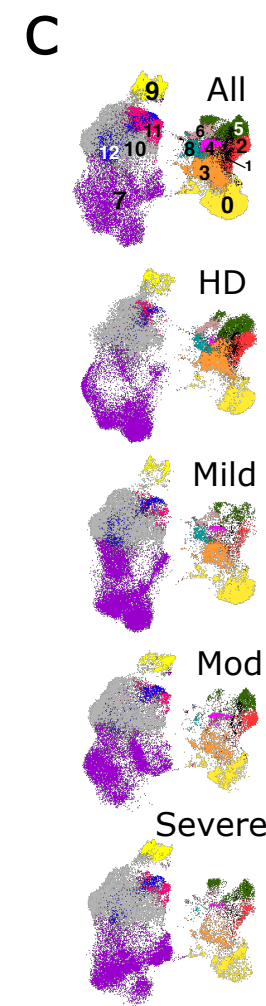

f
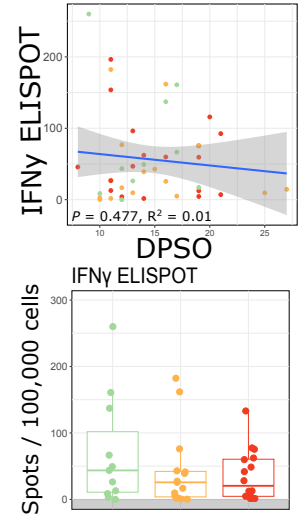

h
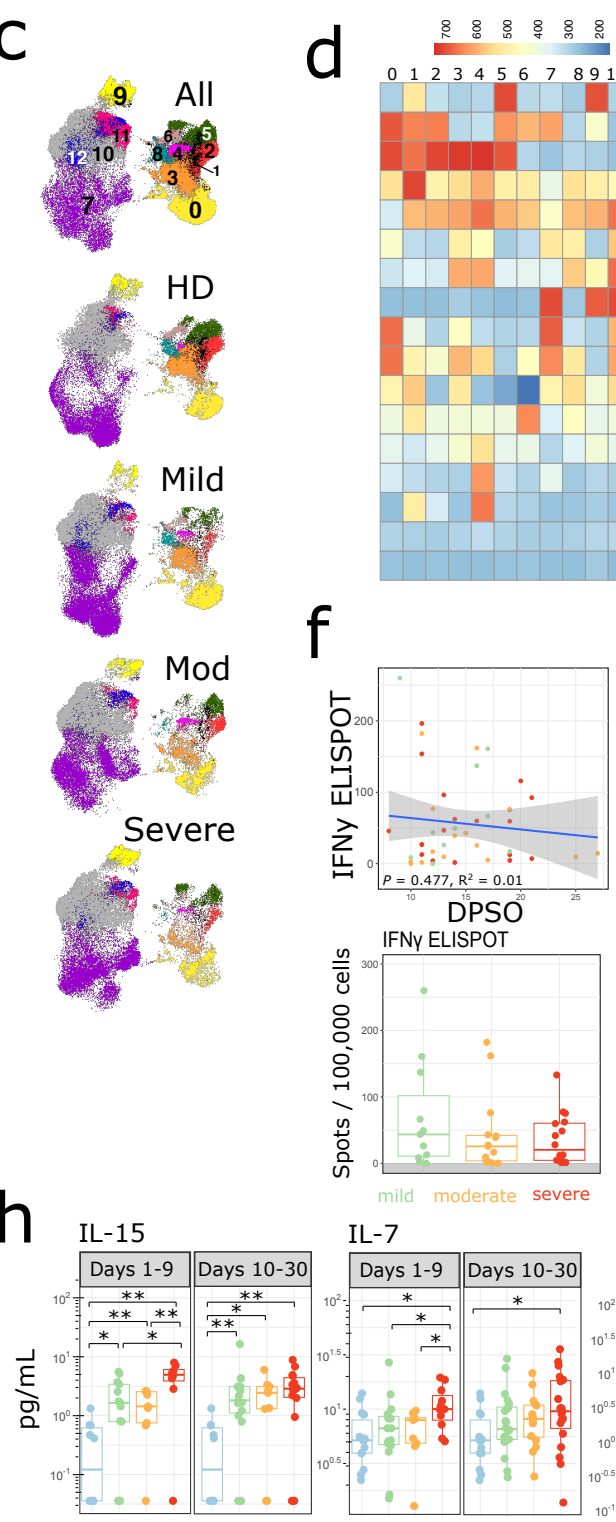

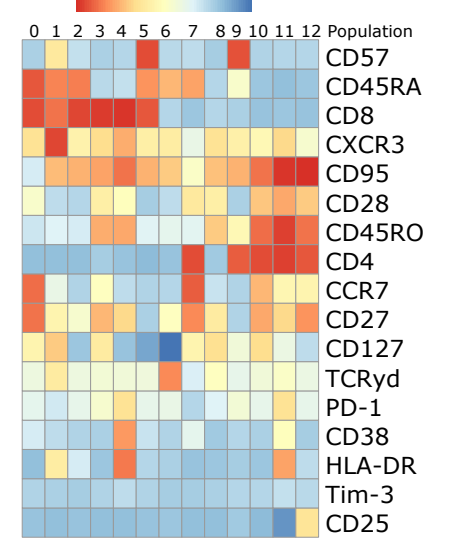

g
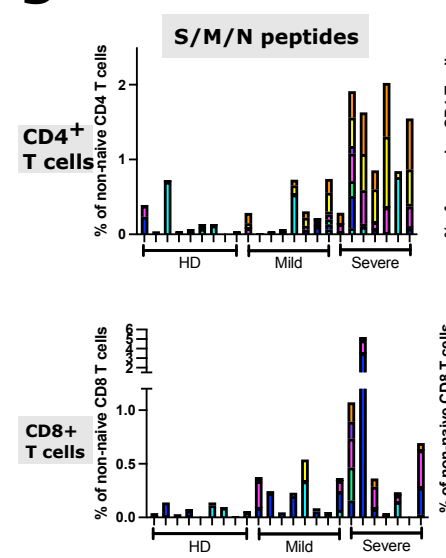
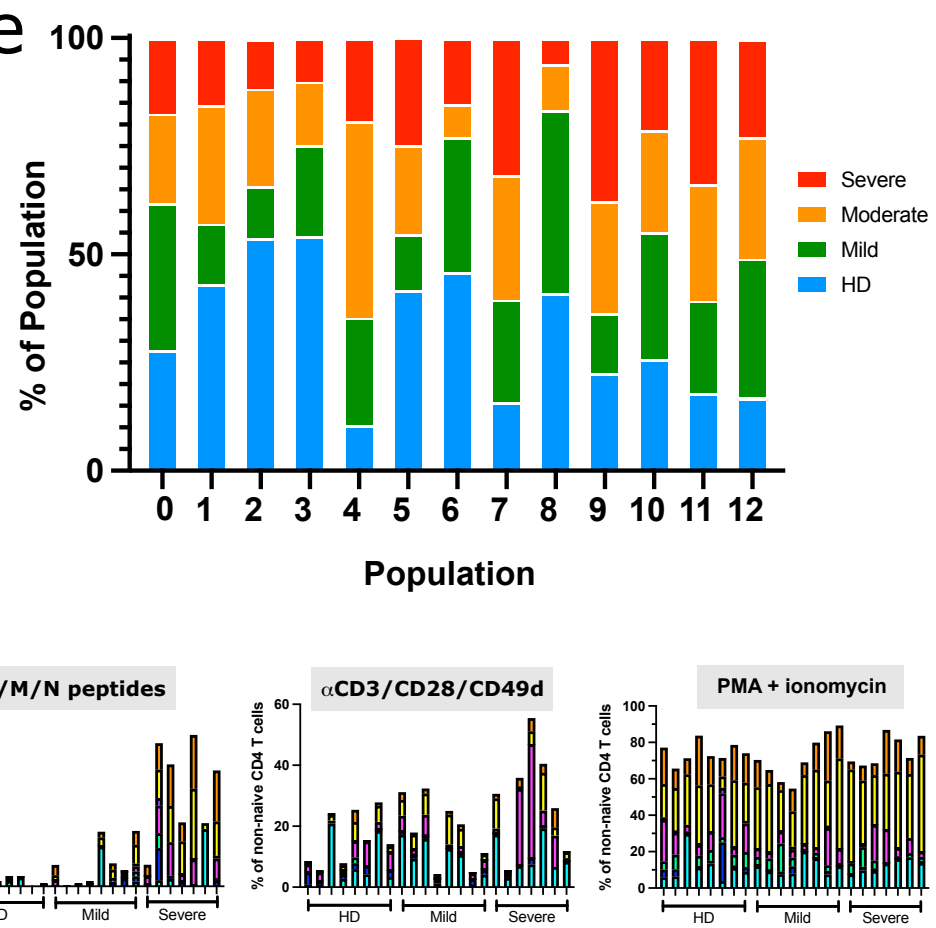

IL-10

\begin{tabular}{|l|l|l|}
\hline Days 1-9 Days 10-30 & Days 1-9 & Days 10-30 \\
\hline
\end{tabular}

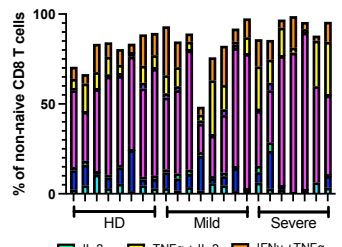

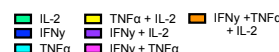

IL-2

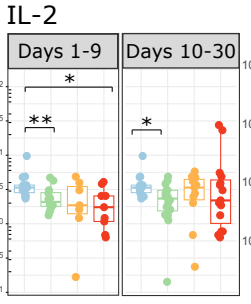


Supplemental Fig 4: SARS-CoV-2 infection is associated with increased activation and functionality of CD4+ and CD8+ T cells. (a-b) Boxplots show MFI of indicated markers in each CD4+ (a) or CD8+ (b) T cell population. Where multiple timepoints within the early (D1-9) or late (D10-30) phase per patient were available, the mean was taken and each patient is represented by one dot per time phase. $n=9$ for $\mathrm{HD}$ (blue), $n=15,6,6$ for mild (green), moderate (orange), and severe (red) respectively in the early phase, and $n=18,15,17$ for mild, moderate and severe in the late phase. (c) UMAP projections of live, CD45+ CD3+ cells from samples between D11-17 DPSO (equal numbers of cells sampled from $n=7,7,7,7 \mathrm{HD}$, mild, moderate, and severe patients, respectively) with FlowSOM clusters overlayed. (d) Marker expression in each FlowSOM cluster shown. (e) The percentage of each cluster derived from HD (blue), mild (green), moderate (orange) or severe (red) patients. (f) PBMCs were stimulated with peptides from the $\mathrm{S}, \mathrm{M}$, or $\mathrm{N}$ proteins in separate wells for 18 hours and IFN- $\gamma$ production measured by ELISPOT. Response to $\mathrm{S}$, $\mathrm{M}$, and $\mathrm{N}$ peptides was summed and normalized per 100,000 cells plated. IFN- $\gamma$ ELISPOT response shown as a linear correlation with DPSO with disease severity indicated by color with shaded area representing $95 \%$ confidence interval (top panel) and as a boxplot by disease severity (bottom panel); $\mathrm{n}=10,17,18$ in mild (green), moderate (orange), and severe (red), respectively. (g) Cytokine production after stimulation with pooled peptides from the S, M and $\mathrm{N}$ proteins, $\mathrm{aCD} 3 / \mathrm{CD} 28 / \mathrm{CD} 49 \mathrm{~d}$ antibodies, or PMA and ionomycin is shown for each individual patient. Background activity in unstimulated wells was subtracted from stimulated wells. The percentage of cells producing various combinations of IFN- $\gamma$, TNFa, and IL-2 were reported. HD: $n=9$ for SMN stimulation, $n=8$ for aCD3/CD28/ CD49d and PMA+ionomycin stimulation; mild $n=8$; severe $n=7$. (h) Maximum cytokine and chemokine levels related to $T$ cell homeostasis are shown during the early phase (days 1-9 from symptom onset) and late phase (days 10-30 from symptom onset) of disease in SARS-CoV-2 infection compared to non-infected healthy controls. Each dot represents maximum value per individual subject during each phase of disease (early phase: mild, $n=15$; moderate, $n=10$; severe, $n=11$ and late phase: mild, $n=23$; moderate, $n=16$; severe, $n=19$ and non-infected healthy controls, $\mathrm{n}=18$ ). ( $\mathrm{a}, \mathrm{b}, \mathrm{f}, \mathrm{h})$ Significance was determined by two-sided Mann Whitney Wilcoxon test and $\mathrm{p}$-values are indicated by asterisks ( $\left.{ }^{*}, p \leq 0.05 ;{ }^{* \star}, p \leq 0.01 ;{ }^{* \star *}, p \leq 0.001,{ }^{* \star \star *}, p \leq 0.0001\right)$. 

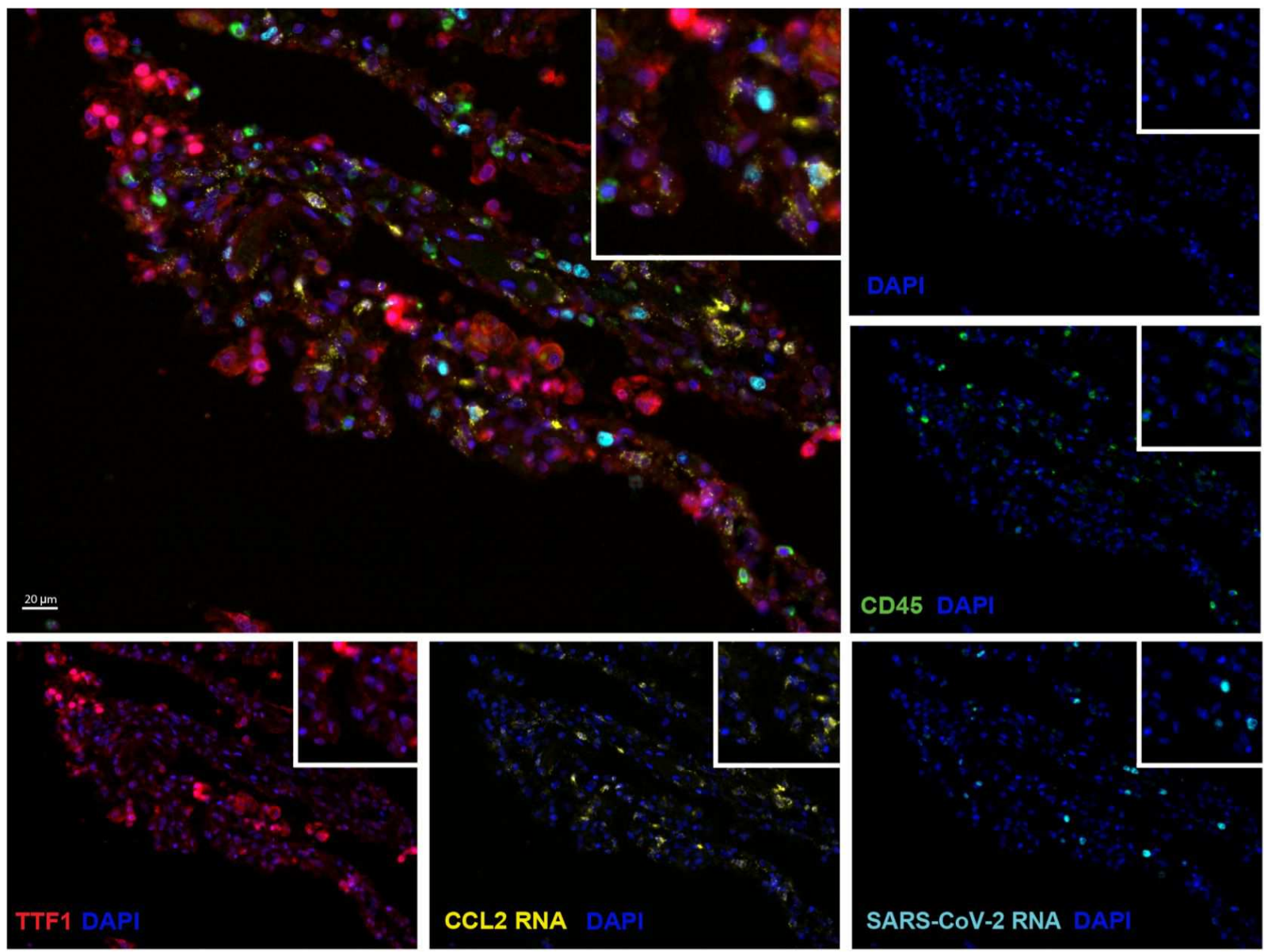

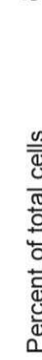

b

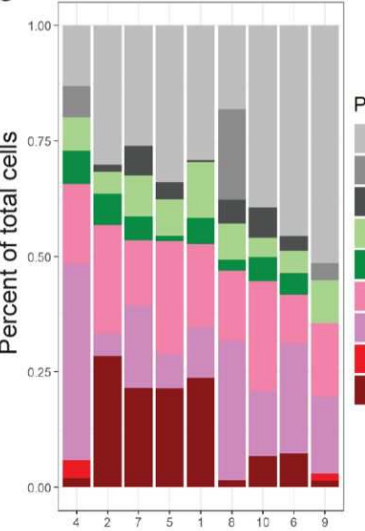

patient ID
C

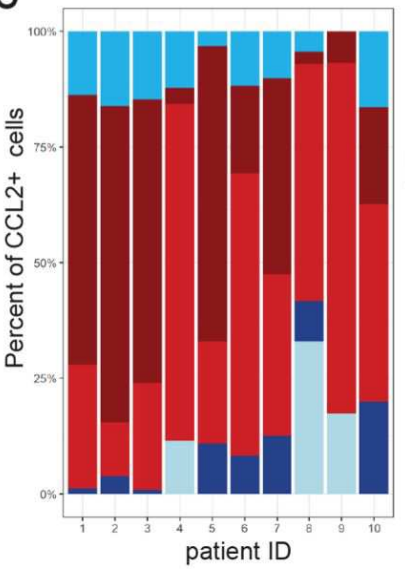

d

Phenotype of CCL2+ cells

CD45

TTF1, SARSCOV2+ TTF1 DAPI, SARSCOV $2+$ DAPI

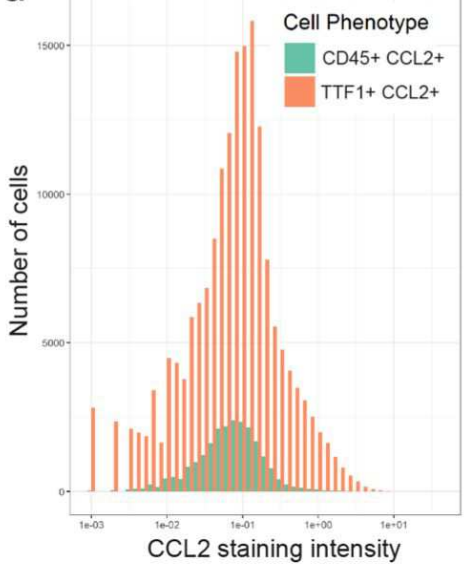

Supplemental Fig 7: Cellular source of CCL2 in autopsy lung tissue in fatal COVID-19. (a) Representative staining for TTF1 (red), CD45 (green), CCL2 RNA (yellow), SARS-CoV-2 RNA (light blue), and nuclear DAPI counterstain (blue); each stain shown separately and merged. Overlaying high-power images showing TTF1+ pneumocytes expressing high levels of CCL2. (b) Bar plots showing the phenotype composition of cell populations in each autopsy lung specimen. (c) Bar plots showing the phenotype composition of CCL2+ cells in each autopsy lung specimen. (d) Histogram displaying the frequency distribution of mean staining intensity for CCL2 between TTF1+ CCL2+ cells (red) versus CD45+ CCL2+ cells (aqua). Cumulative data from all patients shown. 